\title{
Multimodal neuroimaging evidence for looser lexico-semantic networks in schizophrenia:Evidence from masked indirect semantic priming
}

\author{
Gina R. Kuperberg ${ }^{\mathrm{a}, \mathrm{b}, *}$, Kirsten Weber ${ }^{\mathrm{b}, \mathrm{c}}$, Nathaniel Delaney-Busch ${ }^{\mathrm{a}}$, Candida Ustine ${ }^{\mathrm{b}}$, \\ Ben Stillerman ${ }^{\mathrm{b}}$, Matti Hämäläinen ${ }^{\mathrm{b}}$, Ellen Lau ${ }^{\mathrm{b}, \mathrm{d}}$ \\ a Department of Psychology, Tufts University, USA \\ ${ }^{\mathrm{b}}$ Department of Psychiatry and the Athinoula A. Martinos Center for Biomedical Imaging, Massachusetts General Hospital, Harvard Medical School, USA \\ ${ }^{\mathrm{c}}$ Max Planck Institute for Psycholinguistics and the Donders Institute for Brain, Cognition and Behaviour, Radboud University Nijmegen, the Netherlands \\ ${ }^{\mathrm{d}}$ Department of Linguistics, University of Maryland, USA
}

\section{A R T I C L E I N F O}

\section{Keywords:}

MEG

fMRI

N400

Semantic priming

Language

\begin{abstract}
A B S T R A C T
It has been hypothesized that schizophrenia is characterized by overly broad automatic activity within lexicosemantic networks. We used two complementary neuroimaging techniques, Magnetoencephalography (MEG) and functional Magnetic Resonance Imaging (fMRI), in combination with a highly automatic indirect semantic priming paradigm, to spatiotemporally localize this abnormality in the brain.

Eighteen people with schizophrenia and 20 demographically-matched control participants viewed target words ("bell") preceded by directly related ("church"), indirectly related ("priest"), or unrelated ("hammer") prime words in MEG and fMRI sessions. To minimize top-down processing, the prime was masked, the target appeared only $140 \mathrm{~ms}$ after prime onset, and participants simply monitored for words within a particular semantic category that appeared in filler trials.

Both techniques revealed a significantly larger automatic indirect priming effect in people with schizophrenia than in control participants. MEG temporally localized this enhanced effect to the N400 time window (300-500 ms) - the critical stage of accessing meaning from words. fMRI spatially localized the effect to the left temporal fusiform cortex, which plays a role in mapping of orthographic word-form on to meaning. There was no evidence of an enhanced automatic direct semantic priming effect in the schizophrenia group.

These findings provide converging neural evidence for abnormally broad highly automatic lexico-semantic activity in schizophrenia. We argue that, rather than arising from an unconstrained spread of automatic activation across semantic memory, this broader automatic lexico-semantic activity stems from looser mappings between the form and meaning of words.
\end{abstract}

\section{Introduction}

Bleuler proposed that a 'loosening of associations' is a core psychological mechanism underlying multiple features of schizophrenia (Bleuler, 1911/1950). More recently, this mechanism has been conceptualized as an abnormal pattern of automatic activity across lexicosemantic networks (Manschreck et al., 1988; Spitzer et al., 1993). Here we use two complementary neuroimaging techniques - Magnetoencephalography (MEG) and functional Magnetic Resonance Imaging (fMRI) - in combination with a highly automatic indirect semantic priming paradigm to ask when and where this abnormality is localized in the brain, and to shed light on its underlying mechanism.

\subsection{The automatic indirect semantic priming paradigm}

The semantic priming paradigm has provided some of the strongest evidence for abnormal automatic lexico-semantic activity in schizophrenia. The automatic semantic priming effect describes the facilitated processing of a target word (e.g. "bell") when it is very quickly preceded by a prime word that is semantically related (e.g. "church") versus unrelated (e.g. "hammer") to that target. In young, healthy adults, the effect is thought to driven by an automatic bottom-up flow of activity from the prime's word-form representation to its lexical representation, and then to its semantic features as well as those of its

\footnotetext{
* Correspondence to: Department of Psychology, Tufts University, 490 Boston Avenue, Medford, MA 02155, USA.

E-mail address: GKuperberg@mgh.harvard.edu (G.R. Kuperberg).
} 
direct semantic associates, which are stored within semantic memory. If the target word shares some of these pre-activated semantic features, then it is easier to access these features and so its processing is facilitated in comparison with a target that is semantically unrelated to the prime.

An important variant of the semantic priming paradigm is indirect semantic priming. Here, instead of the prime and target being directly related, they are indirectly linked through an unstated mediator word, e.g. "priest" [church] - "bell". In healthy adults, under highly automatic experimental conditions, the indirect semantic priming effect is minimal or absent altogether (de Groot, 1983; McNamara and Altarriba, 1988). This is for two reasons. First, the lexical nodes that describe the mappings between wordform and word meaning are finely tuned such that a particular word-form (e.g. the orthography, p-r-i-e-s-t) maps precisely on to its semantic features and those of its direct associates via a specific lexical representation. Thus, there are no paths through which activity from the word-form (p-r-i-e-s-t) can directly flow to pre-activate either the lexical representation or the semantic features of an indirectly related target (e.g. BELL or $<$ bell $>$ ). Second, in healthy adults, the spread of automatic activity within semantic memory is thought to be constrained and self-limited (perhaps because of inhibitory mechanisms), and so the automatic pre-activation of a prime's directly related semantic features does not, in turn, spread activity to that prime's indirectly related semantic features.

In contrast to healthy adults, people with schizophrenia can show a significant automatic indirect semantic priming effect (e.g. Moritz et al., 2003; Spitzer et al., 1993). This enhanced effect is seen only under automatic conditions and contrasts strikingly with the reduced semantic priming effect that is typically observed in schizophrenia under non-automatic controlled conditions, such as when the interval between the prime and the target (the Stimulus Onset Asynchrony, SOA) is longer, therefore encouraging top-down processing (e.g. Barch et al., 1996). The enhanced automatic indirect semantic priming effect in schizophrenia has been taken as evidence for abnormally broad automatic lexico-semantic activity. It may be linked not only to the symptom of positive thought disorder (the disorganized language output that is seen in a subset of patients), e.g. Spitzer et al., 1993, but more generally to abnormal thought that characterizes schizophrenia as a whole (cf. Bleuler, 1911/1950).

\subsection{Two possible mechanisms of increased automatic indirect priming in schizophrenia}

While the enhanced automatic indirect priming effect in schizophrenia is generally attributed to abnormal automatic lexico-semantic activity, there has been less discussion of precisely how this might arise. Here we outline two possible accounts.

\footnotetext{
${ }^{1}$ By word-form representation, we refer to both the orthographic representation of a word (the way it looks) and the phonological representation of a word (the way it sounds). Most semantic priming studies have presented words visually. In languages such as English, when a word is visually presented, we draw not only upon its orthographic form, but also its phonological form to access its meaning (see Seidenberg and McClelland, 1989). By semantic features, we refer to the set of perceptual features and functional properties that describe the meaning of a given word, e.g. our knowledge that the word, "boy", has the properties of being human, male, young, etc. For simplicity, rather than listing each of these conceptual features for a given word, we use $<>$ around words to refer to this set of conceptual features (e.g. $<$ boy $>$ ). Finally, the term lexical representation has been used in many different ways in the psycholinguistic literature (see Seidenberg, 1990 for discussion). Here we assume a connectionist type architecture (cf Rumelhart et al., 1986) in which lexical representations are conceptualized as nodes that describe the mappings or connections between a particular word-form and a particular set of semantic features. For example, the lexical representation, BOY, describes the connections that map the orthographic word-form, b-o-y, on to the semantic features, $<$ boy $>$.
}

The first is that it reflects an unconstrained spread of activity across semantic memory itself (e.g. Manschreck et al., 1988). This account is depicted diagrammatically in Fig. 1A. In comparison with healthy adults, the spread of automatic activity across semantic memory in people with schizophrenia is less self-limited and broader, perhaps because of reduced inhibitory mechanisms. Thus, when a prime word pre-activates semantic features of its direct semantic associates, this activity spreads further to pre-activate semantic features of its indirect semantic associates. This means that when an indirectly related target word is presented, it is easier to access its semantic features, leading to the indirect semantic priming effect.

Of note, this 'unconstrained spread of semantic activity' account attributes the larger automatic indirect priming effect in schizophrenia to an enhancement of same basic mechanism that is thought to drive the automatic direct priming effect in healthy adults - the spread of activity across semantic memory, which leads to facilitated processing of target words at the semantic level of representation. Because the preactivation of indirectly related semantic features must be mediated by the pre-activation of directly related semantic features, this account predicts not only a larger automatic indirect semantic priming effect in schizophrenia, but also a larger automatic direct priming effect.

In fact, however, the evidence for enhanced automatic direct priming in schizophrenia is less consistent than for enhanced automatic indirect priming. While a few studies report that the automatic direct semantic priming effect is increased in a subgroup of schizophrenia patients with positive thought disorder (Manschreck et al., 1988; Kiefer et al., 2009; Kreher et al., 2008), other studies report no group differences in the direct priming effect (Chapin et al., 1992; Vinogradov et al., 1992; Ober et al., 1995; Barch et al., 1996), even when the same patients show a larger indirect priming effect than controls (e.g. Spitzer et al., 1993).

A second account of increased automatic indirect priming in schizophrenia is that it stems from noisier lexical representations (as noted in footnote 1, we conceive of lexical representations as nodes that describe the mappings or connections between the form and the meaning of words). This account is depicted diagrammatically in Fig. 1B. It holds that these formmeaning (lexical) mappings are weaker and less finely-tuned in people with schizophrenia than in healthy adults (Brown and Kuperberg, 2015; Kuperberg et al., 2018a). For example, in healthy controls, the lexical representation, BELL, encodes tight mappings between the semantic features, $<$ bell $>$, and the specific word-form, b-e-l-l. In schizophrenia, however, the lexical representation, BELL, might encode looser mappings between $<$ bell $>$ and both b-e-l-1 and p-r-i-e-s-t. In the Discussion, we will speculate that such looser lexical mappings might arise as a long-term consequence of sustained abnormal prediction error at the level of wordform and semantic features in schizophrenia. At this stage, we highlight two aspects of this 'noisy lexical representations' account: First, unlike the first account, it provides a direct route through which bottom-up activity can flow from a prime's word-form to pre-activate the representations of its indirect semantic associates, without this necessarily being mediated by the pre-activation of its direct semantic associates. Therefore, this account does not predict that an enhanced automatic indirect priming effect in schizophrenia should necessarily be accompanied by an enhanced direct priming effect. Second, it allows for bottom-up activity to flow from a particular word-form (e.g. p-r-i-e-s-t) to pre-activate the lexical representation of its indirect semantic associate (BELL) without necessarily pre-activating its semantic features. Thus, this account predicts that the enhanced automatic indirect priming effect in schizophrenia can result from facilitated processing of the target at the lexical level of representation (BELL) - not only at the semantic level of representation $(<$ bell $>$ ).

\subsection{Neural evidence for an enhanced automatic indirect semantic priming effect in schizophrenia}

At the neural level, evidence for an enhanced automatic indirect semantic priming effect in schizophrenia comes from studies using event-related potentials (ERPs), which measure neural activity that is 
Two accounts of enhanced automatic indirect priming in schizophrenia A. Unconstrained spread of semantic activity

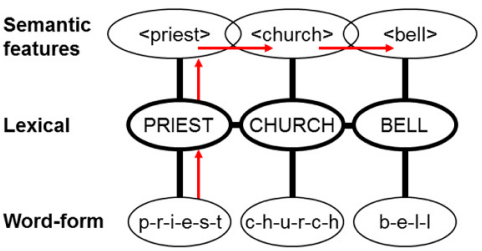

Prime pre-activates indirectly related semantic features

B. Noisier lexical representations

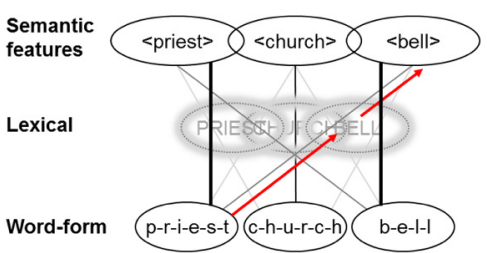

Prime pre-activates indirectly related semantic features

Prime pre-activates noisy lexical representations

Fig. 1. Diagrammatic illustration (highly simplified) of two accounts of an enhanced automatic indirect priming semantic effect in schizophrenia. Word knowledge is depicted at three levels of representation: word-form (here, illustrated as orthographic form), semantic features (here, we use $<>$ around a word to indicate a set of conceptual features associated with that word), and lexical representations, which describe the mappings/connections that link semantic features and word-form. Solid lines are used to indicate these mappings/ connections, with the thickness of the lines depicting the strength of these connections. Red arrows are used to indicate the flow of information after encountering a prime word, "priest". A illustrates an unconstrained spread of activity across semantic memory in schizophrenia. Just as in healthy adults, the lexical representations are precise - that is, the mappings between the meaning of a particular word and its word-form are strong (depicted as thick lines) and finely-tuned (1:1 mappings). However, activity spreads further across semantic memory than in healthy controls. Thus, in healthy controls, after encountering a particular prime word (e.g. "priest"), activity flows from the prime's form (here depicted as p-r-i-e-s-t) to its specific lexical representation (PRIEST), and to its semantic features ( $<$ priest $>$ ) as well as directly associated semantic features $(<$ church $>$ ). In schizophrenia, the pre-activation of the directly associated semantic features ( $<$ church $>$ ), in turn, spreads activity to the semantic features of its own associates (e.g. $<$ bell $>$ ). This flow of activity is depicted with red arrows. This means that if the indirectly related target word, "bell", appears very quickly after the prime, its processing will be facilitated at the level of semantic features, leading to an enhanced automatic indirect semantic priming effect. B illustrates noisier lexical representations in schizophrenia. The mappings between semantic features and word-form are weaker (depicted as thinner/fainter lines) and less finely-tuned (no 1:1 mappings) than in healthy adults, perhaps as a long-term consequence of adapting to sustained abnormal prediction error at the level of word-form and semantic features (see Discussion). Here, the lexical representation, BELL, encodes mappings between $<$ bell $>$ and both b-e-l-1 and p-r-i-e-s-t. Thus, after encountering the prime word, "priest", there is a direct route through which bottom-up activity can flow from its orthographic form (p-r-i-e-s-t) to pre-activate its indirectly related lexical representation (BELL) and its indirectly related semantic features $(<$ bell $>$ ). This flow of activity is depicted with red arrows. This means that if the indirectly related target word, "bell", appears very quickly after the prime, its processing can be facilitated at both the lexical level and the semantic level. (For interpretation of the references to color in this figure legend, the reader is referred to the web version of this article.)

time-locked to the onset of the target. Under semi-automatic conditions, people with schizophrenia show more neural facilitation to indirectly related target words than healthy controls on the N400 (Kreher et al., 2008; Kreher et al., 2008; Mathalon et al., 2002) - an ERP that is evoked between 300 and $500 \mathrm{~ms}$ and that indexes facilitated semantic processing (Kutas and Federmeier, 2011). ERPs, however, do not have the necessary spatial resolution to determine where this enhanced semantic facilitation to indirectly related targets is localized in the brain.

Functional Magnetic Resonance Imaging (fMRI) does have the necessary spatial resolution to address this question. However, no fMRI study to date has shown evidence of an enhanced automatic indirect semantic priming effect in schizophrenia. In healthy individuals, fMRI studies of automatic direct semantic priming have reported modulation in two regions within the temporal cortex. The first is the anterior portion of the left lateral temporal cortex. This region is thought to play an important role in accessing the semantic features of incoming words, acting as a hub that 'collects together' semantic information that is widely distributed across the cortex (Lambon Ralph and Patterson, 2008; Lambon Ralph et al., 2017). In a recent study of masked highly automatic direct semantic priming in young healthy adults, we used multimodal imaging techniques (ERP, MEG and fMRI) to show that this region was modulated by priming within the N400 time window (Lau et al., 2013).

The second region where direct automatic semantic priming effects have been reported in healthy adults is the left temporal fusiform cortex, located on the ventromedial surface of the temporal lobe (e.g. Wheatley et al., 2005; Gold et al., 2006). This region is particularly likely to be modulated during direct priming when participants carry out tasks like lexical decision, which encourage lexical rather than semantic processing. It is thought to mediate lexical processing at the interface between accessing pure orthographic representations in the posterior occipital fusiform cortex and semantic features within the lateral anterior temporal cortex (Price and Devlin, 2011).

In schizophrenia, only one previous fMRI study has attempted to probe automatic semantic priming by using a short SOA of $350 \mathrm{~ms}$ between prime and target (Wilson et al., 2013). This study reported no significant difference between patients and controls in direct priming, but a significant between-group difference in indirect priming, which localized to the left fusiform cortex. However, instead of this difference being driven by a larger indirect priming effect in the schizophrenia group (that is, reduced activity to the indirectly related versus the unrelated word-pairs), it was driven by a reverse hemodynamic priming effect in the schizophrenia group - more activity to the indirectly related than the unrelated word-pairs. This type of reversed hemodynamic priming effect within fusiform cortices in people with schizophrenia has previously been reported in fMRI studies that used a longer SOA between prime and target, therefore biasing towards non-automatic controlled processing (Han et al., 2007; Kuperberg et al., 2007). These previous studies also reported a reverse priming effect in prefrontal cortices, which are known to mediate controlled top-down semantic activity (Gold et al., 2006; Thompson-Schill et al., 2005; Kuperberg et al., 2008). Both reverse hemodynamic priming effects were attributed to inefficient top-down controlled processing in schizophrenia; the assumption was that, integrated over the sluggish hemodynamic response, inefficient top-down processes led to prolonged lexico-semantic matching processes within temporal cortices to indirectly related (versus unrelated) word-pairs, which outweighed any effects of automatic facilitation (Kuperberg et al., 2007).

We suggest that in Wilson et al's (2013) study, despite the short SOA between the prime and the target, the use of a lexical decision task encouraged top-down controlled processing, which drove the reverse hemodynamic indirect priming effect. Lexical decision is known to encourage controlled semantic matching processes (Neely, 1991), and semantic matching is known to reduce the semantic priming effect in schizophrenia, even at short SOAs (Kreher et al., 2009, Explicit task). Indeed, a previous ERP study using a lexical decision task with a short SOA reported a reduced (rather than an increased) N400 indirect priming effect in schizophrenia (Kiang et al., 2008).

\subsection{The present study}

In the present study, we used two complementary neuroimaging techniques - fMRI and Magnetoencephalography (MEG) - to spatiotemporally localize the effects of highly automatic indirect priming in schizophrenia under experimental conditions that minimized or 
eliminated top-down controlled processing altogether. We used an SOA that was shorter than that used in previous studies of automatic priming in schizophrenia $(140 \mathrm{~ms}$ ), and we masked the prime word (sandwiching it between strings of pound signs) such that it was perceived just over the threshold of awareness (Marcel, 1983). In addition, instead of carrying out a lexical decision on each trial, which can encourage top-down processing, participants monitored for words within a particular semantic category (insects), which appeared only in filler trials (Kreher et al., 2006; Misra and Holcomb, 2003). People with schizophrenia and demographically matched control participants viewed exactly the same set of stimuli in both fMRI and MEG sessions, with stimuli being fully counterbalanced across sessions.

Our primary goal was to seek neural evidence of an enhanced automatic indirect priming effect in schizophrenia. Finding such an effect using both MEG and fMRI techniques in the same set of participants would provide strong and converging neural evidence for abnormally broad automatic lexico-semantic activity. In MEG, we hypothesized that an enhanced highly automatic indirect priming effect in schizophrenia would manifest within the N400 time window. This would build upon and extend previous ERP studies that have reported enhanced indirect priming in schizophrenia under semi-automatic (nonmasked) conditions (e.g. Kreher et al., 2008). In fMRI, we hypothesized that the enhanced automatic indirect hemodynamic priming effect would localize to the temporal cortex, in the absence of prefrontal modulation. This would provide the first fMRI evidence for enhanced automatic indirect priming in schizophrenia.

We also aimed to gain insights into the mechanism underlying any enhanced automatic indirect priming effect in schizophrenia. As discussed above, the 'unconstrained spread of semantic activity' account predicts that we should see both an enhanced direct and indirect priming effect in schizophrenia. Given that priming is thought to be driven by facilitation at the level of semantic features, it also predicts that both these effects should localize to the anterior lateral temporal cortex - the region that is thought to play a role in accessing semantic features (Lambon Ralph and Patterson, 2008; Lambon Ralph et al., 2017), and where our previous multimodal imaging study (which used the same masked priming paradigm in a younger group of healthy controls) localized the automatic direct priming effect within the N400 time window (Lau et al., 2013).

In contrast, the 'noisy lexical representations' account allows for an enhanced automatic indirect priming effect in schizophrenia without this necessarily being accompanied by an enhancement of the automatic direct priming effect. It also holds that an enhanced automatic indirect priming effect can result not only from facilitated processing of the target's semantic features, but also from facilitated access to its lexical representation. It therefore predicts that we should see an enhanced indirect priming effect in schizophrenia within the left (temporal) fusiform cortex, which is thought to play a role in lexical processing at the interface between word-form and meaning (Price and Devlin, 2011), and where previous fMRI studies of indirect semantic priming in schizophrenia, carried out under less automatic conditions, have reported abnormal modulation (Kuperberg et al., 2007; Han et al., 2007; Wilson et al., 2013).

\section{Methods}

\subsection{Materials}

The stimulus materials have previously been described in detail (Kreher et al., 2006; Lau et al., 2013). They constituted 192 unique targets (e.g. "bell") paired with primes that were either directly related (e.g. "church"), indirectly related (e.g. "priest"), or unrelated (e.g. "hammer"). As previously described (Kreher et al., 2006), the primes of the indirectly related pairs did not share obvious categorical relationships or direct associations with their targets. However, they did share more global similarities with their targets than the unrelated word-pairs, as indexed by Latent Semantic Analysis (Landauer and Dumais, 1997; Landauer et al., 1998; see also Chwilla and
Kolk, 2002), as well as by a norming study in which participants were explicitly asked to identify the unstated mediator word (described by Kreher et al., 2006, pages 553 and 556-557).

The same targets were counterbalanced across three lists. Each list contained 64 directly related pairs, 64 indirectly related pairs, and 64 unrelated pairs, in randomized order. No prime or target word appeared twice in the same list, but across all participants, the same targets could appear in all three conditions. Thus, differences between conditions could not be attributed to differences to the lexical properties of the targets or the primes.

Each list also contained 40 filler unrelated word-pairs in which either the prime $(50 \%)$ or the target $(50 \%)$ was the name of an insect. These were included for the purpose of the semantic monitoring task (described below).

\subsection{Participants}

People with a DSM-IV diagnosis of schizophrenia or schizoaffective disorder (confirmed with a Structured Clinical Interview, see Spitzer et al., 1992) were recruited from the Lindemann Mental Health Center, Boston. Demographically matched volunteers without histories of psychiatric disorders were recruited by advertisement. All participants were right-handed, as assessed using the Edinburgh handedness questionnaire (Oldfield, 1971), and were native, primarily monolingual American English speakers. Participants were excluded if they had a history of neurological injury, medical disorders impairing neurocognitive function, or if they met DSM-IV criteria for substance abuse (within three months) or substance dependence. Written consent was obtained following the guidelines of the Massachusetts General Hospital Human Subjects Research Committee. Most patients' symptoms were assessed on the day of either the fMRI or the MEG session using the Scale for the Assessment of Positive Symptoms (SAPS: Andreasen, 1984a), and the Scale for the Assessment of Negative Symptoms (SANS: Andreasen, 1984b).

Our control dataset was $n=20$ for fMRI and $n=18$ for MEG. Twenty-one control participants were originally recruited. One was subsequently excluded because of a structural MRI abnormality, and two failed to return for the MEG session. Our schizophrenia dataset was $\mathrm{n}=16$ for fMRI and $\mathrm{n}=18$ for MEG. Twenty-two schizophrenia participants were originally recruited, but two were excluded from both fMRI and MEG analysis (technical problems and a failure to complete the fMRI study). Four additional patients were excluded from the fMRI analysis: two because more than $20 \%$ of volumes yielded evidence of artifact as detected using the ArtRepair toolbox (Mozes and WhitfieldGabrieli, 2011; for further details, see fMRI analysis below), one because of a susceptibility artifact on the functional scans (of unknown origin), and one because of poor behavioral performance. In addition, two patients failed to return for the MEG session.

In our analyses of the MEG and fMRI data, we included all participants who completed that session. Of these participants, 14 control participants and 18 people with schizophrenia participated in both fMRI and MEG sessions. We show the demographic and clinical data of this subset of participants in Table 1 . The two groups were matched closely on gender, race, and ethnicity, all ps $>0.1$, and on age, years of education, and parental socioeconomic status (Hollingshead, 1965), all ps $>$ 0.3. Premorbid verbal IQ, assessed with the North American Adult Reading Test (Blair and Spreen, 1989), was slightly lower in the patient than the control group $(\mathrm{t}(30)=2.103, \mathrm{p}=0.044)$.

\subsection{Overall procedure}

For the subset of participants who completed both MEG and MRI sessions, these sessions were carried out on different days, with a minimum of two days in between sessions. The order of these sessions was counterbalanced across participants. In addition, participants were assigned different lists in each session so that no participant saw the same list (or target word) twice. 
Table 1

Demographic and symptom measures of the subset of participants whose data were included in both fMRI and MEG analyses.

\begin{tabular}{|c|c|c|}
\hline & $\begin{array}{l}\text { Controls } \\
(n=18)\end{array}$ & $\begin{array}{l}\text { Schizophrenia } \\
(n=14)\end{array}$ \\
\hline Gender $(\mathrm{F} / \mathrm{M})$ & $3 / 15$ & $3 / 11$ \\
\hline $\begin{array}{l}\text { Race (Asian/Native Hawaiian or Other } \\
\text { Pacific Islander/Black/White) }\end{array}$ & $1 / 0 / 8 / 9$ & $0 / 0 / 2 / 12$ \\
\hline Age (years) & $45.2(6.99)$ & $44.4(9.48)$ \\
\hline Hollingshead Index ${ }^{\mathrm{a}}$ & $2.78(0.81)$ & $2.50(0.86)$ \\
\hline Premorbid Verbal $\mathrm{IQ}^{\mathrm{b}}$ & $110(8.3)$ & $103(11.8)$ \\
\hline CPZ Equivalent ${ }^{\mathrm{c}}$ & - & $518(262)$ \\
\hline Duration of illness (years) & - & $18.9(9.06)$ \\
\hline SAPS $^{\mathrm{d}}$ & - & $4.07(3.77)$ \\
\hline SANS $^{\mathrm{e}}$ & - & $3.64(3.77)$ \\
\hline $\begin{array}{l}\text { Anticholinergics: receiving/not } \\
\text { receiving }\end{array}$ & - & $2 / 12$ \\
\hline Typical/Atypical antipsychotics & - & $2 / 12$ \\
\hline
\end{tabular}

Means are shown with standard deviations in parentheses. M: Male; F: Female.

a Parental socio-economic status (SES) was calculated using the Hollingshead Index (Hollingshead, 1965).

b Premorbid IQ was assessed using the North American Adult Reading Test: NAART (Blair and Spreen, 1989).

c Chlorpromazine (CPZ) Equivalents were calculated following the International Consensus Study of Antipsychotic Dosing (Gardner et al., 2010).

d SAPS: Scale for Assessment of Positive Symptoms (Andreasen, 1984a, 1984b); the sum of the global scores is reported.

e SANS: Scale for the Assessment of Negative Symptoms (Andreasen, 1984a, 1984b); the sum of the global scores is reported.

\subsection{Stimuli presentation and task}

The structure of trials is shown in Fig. 2. For fMRI, additional fixations (2-8s) were added after $25 \%$ of trials to optimize the deconvolution of the event-related hemodynamic response function (Burock and Dale, 2000; see https://surfer.nmr.mgh.harvard.edu/ optseq). For MEG, at least $700 \mathrm{~ms}$ was inserted between trials, allowing participants time to blink. In both sessions, participants carried out a semantic monitoring task: to press a response button as quickly as possible whenever they saw an insect word, regardless of whether it appeared in the prime or target position. Responses were considered accurate if they were registered within $5 \mathrm{~s}$ of prime onset. Trials were presented in two runs, separated by a short break. A brief practice session preceded both the MEG and fMRI sessions.

\section{5. $M E G$ acquisition}

MEG data was acquired inside a magnetically shielded room (IMEDCO AG, Switzerland) using a Neuromag VectorView system (Elekta-Neuromag Oy, Finland; 102 sets of triplet sensors - two orthogonal planar gradiometers and one magnetometer), with an online bandpass filter of $0.03-200 \mathrm{~Hz}$ and continuously sampled at $600 \mathrm{~Hz}$. We also collected EEG, EOG, and ECG data using a 70-channel MEG-compatible electrode system. The EEG data are not reported here. To record the position and orientation of the head with respect to the MEG sensor array, at the beginning of each run, the locations of three fiduciary points (nasion and two auricular), four head position indicator coils, the EEG electrodes, and $100+$ additional points were digitized using a 3Space Fastrak Polhemus digitizer integrated with the Vectorview system.

\subsection{FMRI acquisition}

fMRI images were acquired using a 3T Siemens Trio scanner and a 32-channel head coil using a gradient-echo sequence (TR, $2 \mathrm{~s}$; TE, $25 \mathrm{~ms}$; flip angle $90^{\circ}$; interleaved acquisition) over two runs (each 148 functional volumes; 36 axial slices anterior commissure-posterior commissure aligned, $3 \mathrm{~mm}$ slice thickness, $0.3 \mathrm{~mm}$ skip, $200 \mathrm{~mm}$ field of

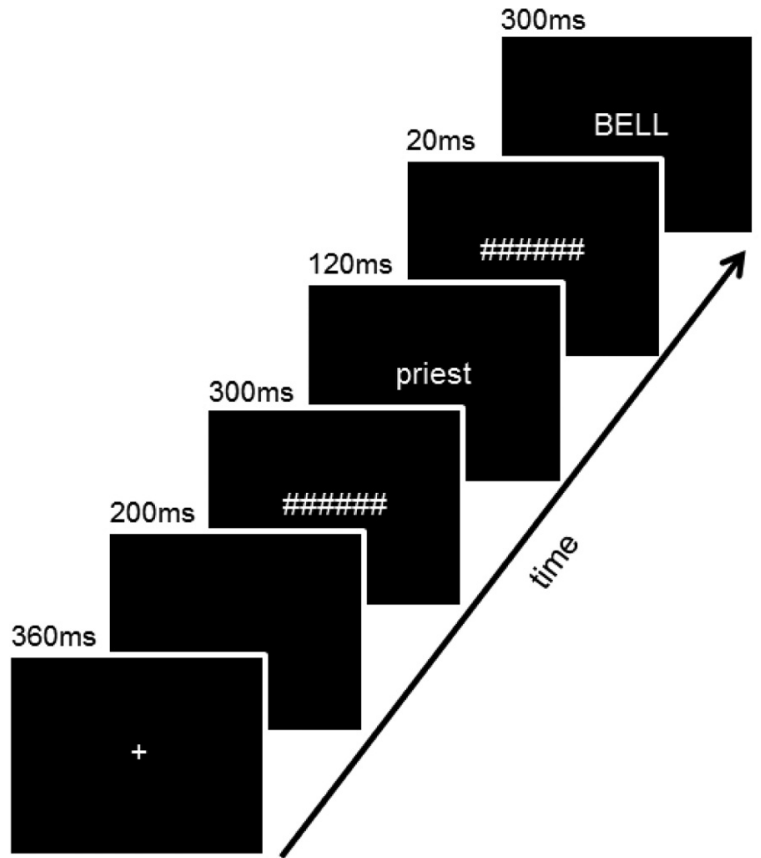

Fig. 2. Stimulus presentation sequence, illustrated with an example of a semantically indirectly related prime-target pair. Each trial lasted for $1300 \mathrm{~ms}$ and constituted a central fixation for $360 \mathrm{~ms}$, a blank screen for $200 \mathrm{~ms}$, a forward mask (a hash mark string the length of the longest prime word) for $300 \mathrm{~ms}$, the prime word (lower case) for $120 \mathrm{~ms}$, a backward mask for $20 \mathrm{~ms}$, and finally the target word (upper case) for $300 \mathrm{~ms}$. This resulted in a stimulusonset asynchrony of $140 \mathrm{~ms}$.

view, in-plane resolution of $3.125 \mathrm{~mm}$ ). We also acquired structural images at the beginning and the end of the session.

\subsection{MEG preprocessing}

Signal-space projection (SSP) was applied to MEG magnetometer data to suppress environmental noise and biological artifacts (Uusitalo and Ilmoniemi, 1997). Additional SSPs were computed to estimate cardiac artifacts (detected using the bipolar ECG electrodes) and ocular artifacts (detected using the bipolar VEOG electrodes) and applied to the MEG gradiometer and magnetometer data. Averaged event-related MEG signals were computed off-line from trials free of muscular artifacts after the application of a $20 \mathrm{~Hz}$ off-line low-pass filter. We then combined activity across the two planar gradiometers at each site, and calculated activity timelocked to target words in each condition using a baseline of $-100 \mathrm{~ms}$. We generated difference waveforms corresponding to the indirect and direct priming effects, and averaged these difference waveforms across 13 temporal sites where previous MEG studies have reported maximal modulation within the N400 time window (e.g. Halgren et al., 2002; Helenius et al., 1998; Lau et al., 2013).

\subsection{MEG statistical analysis}

To determine whether the indirect or the direct priming effects differed between the schizophrenia and control groups, we used a mass univariate approach: We carried out independent-samples $t$-tests (comparing the two groups) at each sampling point (60 data points) on the difference waveforms (unrelated minus directly related; unrelated minus indirectly related) within the $300-500 \mathrm{~ms}$ time window of interest, averaged over the temporal sites. We used cluster-based permutation tests to account for multiple comparisons (Maris and Oostenveld, 2007; Groppe et al., 2011) using an open-source Matlab toolbox within the Fieldtrip software package (Oostenveld et al., 2011). Consecutive data points that exceeded a pre-set uncorrected 

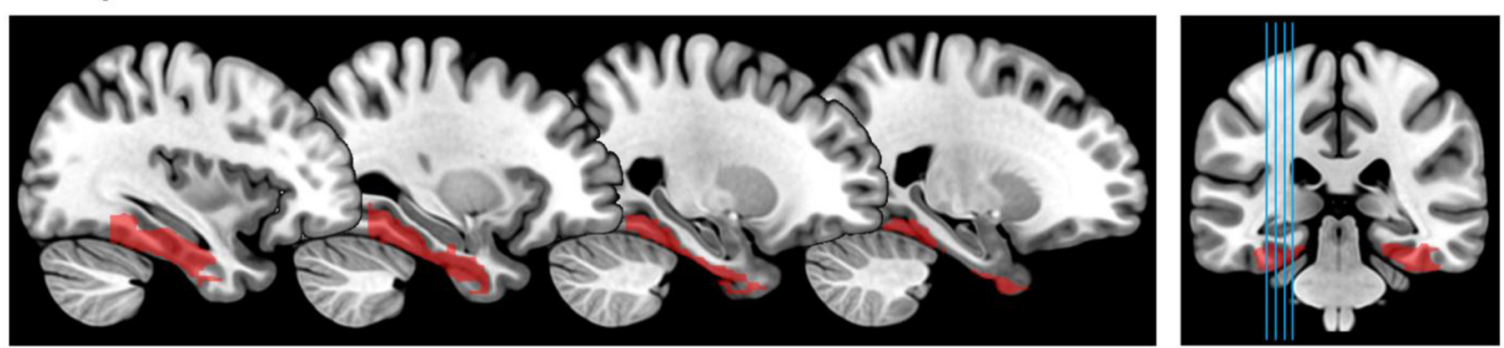

\section{Left anterior superior/middle temporal cortex}
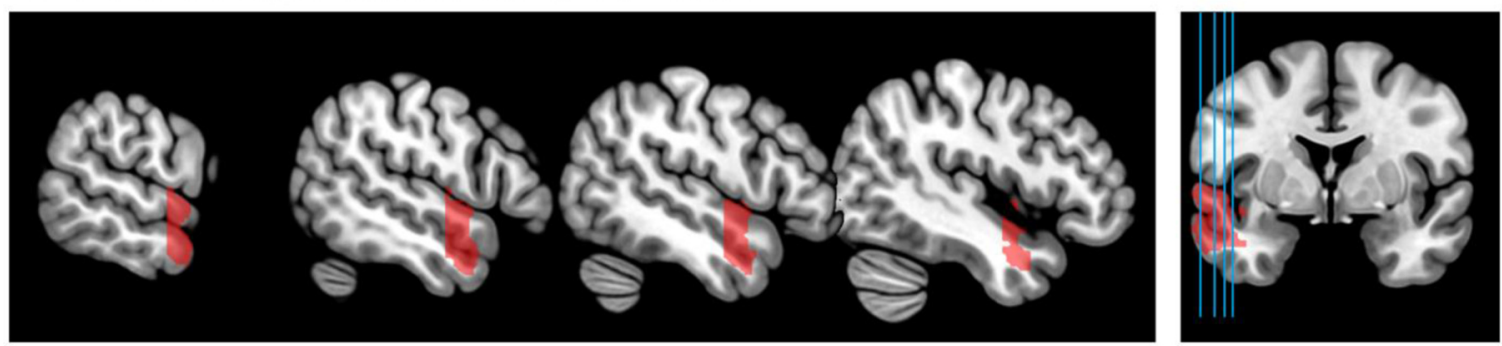

Fig. 3. Anatomical Regions of Interest (ROIs) used for small volume correction. These were defined using the Anatomical Automatic Labeling (AAL) regions of interest (Tzourio-Mazoyer et al., 2002), with coronal planes specifying posterior-anterior boundaries recommended by Rademacher et al. (1992). They were created with the MarsBaR toolbox in SPM (Brett et al., 2002). Top row: The temporal fusiform ROI. This constituted both right and left fusiform gyri (defined using the AAL template), constrained posteriorly by the occipito-temporal junction. The position of the occipito-temporal junction was determined by visual inspection of the ch2better.nii.gz template brain in mricron and operationalized as the region anterior to a coronal plane located at y-coordinate -55 in MNI space. Left: sagittal slices at $-35,-30,-26$ and -22 ; Right: Coronal slice at $y=-33$, with blue lines indicating the position of the sagittal slices. Bottom row: The left anterior superior/ middle temporal ROI. This constituted both the left superior and middle temporal gyri (defined using the AAL template), constrained posteriorly by a coronal pane from the rostrolateral end of the first transverse sulcus, located at y-coordinate -7 in MNI space. Left: sagittal slices at $-61,-54,-50$ and -46 ; Right: Coronal slice at $y=0$, with blue lines indicating the position of the sagittal slices. (For interpretation of the references to color in this figure legend, the reader is referred to the web version of this article.)

significance threshold of $5 \%$ were considered a cluster, and individual tstatistics within each cluster were summed to yield cluster-level test statistics. Next, a null distribution was created by randomly assigning difference values at each sampling point to either the schizophrenia or the control group 1000 times, calculating cluster-level statistics for each randomization and entering the largest cluster-level summed $t$ statistic into the null distribution (Maris and Oostenveld, 2007). The actually observed cluster-level test statistics were compared with the null distribution, and any clusters falling within the highest or the lowest 2.5th percentile were considered significant.

To follow up any significant between-group differences, we examined priming effects within each group separately by carrying out dependent sample $t$-tests between conditions at each sampling point between 300 and $500 \mathrm{~ms}$, and once again using a cluster-based permutation approach to account for multiple comparisons.

\section{9. $f M R I$ preprocessing}

Analysis of the fMRI data was conducted in SPM8 (www.fil.ion.ucl. ac.uk/spm). After discarding the first four images, spikes and other artifacts were detected. Bad slices (average: 0.5\%; range: 0-7.6\%) were interpolated from surrounding images using the ArtRepair toolbox (Mazaika et al., 2009). In each participant, images were then slice-time corrected and realigned to the first image of each run and then to each other. The mean of the functional images was co-registered to the structural images. The structural images were segmented into gray and white matter and the functional images were spatially normalized and then smoothed with a $10 \mathrm{~mm}$ FWHM Gaussian kernel. The selection of this smoothing kernel was based on a previous semantic priming fMRI in healthy adults (Weber et al., 2016); it is appropriate for detecting small effects (such as the masked priming effect) at the group level, while still accommodating anatomical and functional variation between individuals (see Mikl et al., 2008 for discussion) - a particularly important consideration in schizophrenia (e.g. Gopal et al., 2016).

We next used the Artifact Detection Toolbox (ART, Mozes and Whitfield-Gabrieli, 2011) to calculate the percentage of time points/ volumes (across all runs) in which either the composite motion was greater than $1 \mathrm{~mm}$ and/or the image intensity was greater than $6 * \mathrm{SD}$ of the mean image intensity of that participant. Participants in which more than $20 \%$ of volumes met one or both of these two criteria were excluded (as noted above, this resulted in the exclusion of two people with schizophrenia). In the remainder of participants, we used the toolbox to create additional regressors that identified any volume that met either or both of the above criteria. Across these remaining participants, less than $2 \%$ of volumes/time points were marked.

At the first level of analysis, each run was modeled with a design matrix that included regressors for each of the three experimental conditions and the two types of filler trials (insect word in the prime and the target position). Trials were modeled from $100 \mathrm{~ms}$ before trial onset until the end of the trial (event duration: $1400 \mathrm{~ms}$ ) and regressors were convolved with a canonical hemodynamic response function. The model also included the additional nuisance regressors created using the Artifact Detection Toolbox as described above. We defined three contrasts to take to the second level: (a) unrelated word-pairs versus implicit baseline, (b) indirectly related word-pairs versus implicit baseline, (c) directly related word-pairs versus implicit baseline.

\subsection{0. fMRI statistical analysis}

At the group level, our main focus was on two a priori Regions of Interest (ROIs) which we used for small volume correction - bilateral temporal fusiform cortex (temp-fusiform) and the left anterior superior/ middle temporal cortex (left ant-S/MTG), see Fig. 3 for full description. We carried out two separate ANOVAs that crossed Group 
Table 2

Accuracy in identifying probe words during the semantic monitoring task in the MEG and fMRI sessions. Means are shown with standard deviations in parentheses.

\begin{tabular}{lcclcc}
\hline & \multicolumn{2}{c}{ Control group } & & \multicolumn{2}{c}{ Schizophrenia group } \\
\cline { 2 - 3 } \cline { 5 - 6 } & MEG & fMRI & & MEG & fMRI \\
\hline Prime position & $0.52(0.26)$ & $0.62(0.26)$ & & $0.27(0.27)$ & $0.47(0.33)$ \\
Target position & $0.86(0.15)$ & $0.89(0.14)$ & & $0.74(0.16)$ & $0.83(0.15)$ \\
\hline
\end{tabular}

(schizophrenia, control) and Relatedness (indirectly related vs. unrelated, or directly related vs. unrelated) and constructed Statistical Parametric Maps (SPMs) to reveal any voxels that showed a significant Group by Relatedness interaction, (i.e. a between-group difference in either the indirect priming effect or the direct priming effect). For these analyses, we set an initial voxel-level threshold of $\mathrm{p}<0.005$ (whole brain), and we inferred significance if the peak voxel within the ROI reached a familywise error-corrected (FWE) threshold of $\mathrm{p}<0.05$ using a small volume correction (Worsley et al., 1996). We report the MNI coordinates, $\mathrm{z}$ score and $\mathrm{p}$ value of this peak. To follow up any Group by Relatedness interaction, we averaged across all voxels within the ROI that were activated at the $\mathrm{p}<0.005$ whole brain voxel-wise threshold, and we carried out two-tailed $t$-tests to examine the effect of Relatedness in each group separately.

In addition to these $a$ priori analyses, we also carried out exploratory whole brain analyses. First, we examined the direct and indirect priming effects in the schizophrenia and the control groups separately. Second, we compared the contrast between all word-pairs and the implicit baseline between the schizophrenia and the control groups. In these exploratory whole brain analyses, we used a liberal initial voxellevel threshold of $p<0.05$. This is because their main purpose was to determine whether there were any other regions that were modulated outside our regions of interest, even at a liberal threshold. We report any cluster that was significant at a cluster-level FWE-corrected threshold of $\mathrm{p}<0.05$. We report the size and the $\mathrm{p}$ value of the cluster (as a whole) as well as the $\mathrm{z}$ scores and uncorrected $\mathrm{p}$ values of the individual peaks within that cluster. All coordinates reported are in MNI space.

\section{Results}

\subsection{Behavioral results}

Accuracies for identifying insect word probes in the semantic monitoring task are shown in Table $2 .^{2}$ In both MEG and fMRI sessions, both groups identified probes in the target position more accurately than in the prime position (main effects of Probe Position: Fs $>57$; $p s<0.0001$ ). In the MEG session, patients were less accurate than controls, collapsed across prime and target positions (main effect of Group: $F(1,34)=9.583, p<0.01$ ), but in the fMRI session, there was no difference between the two groups (main effect of Group: $F(1,30)$ $=1.388, p>0.2$ ). There was no difference between patients and controls in their differential accuracies in identifying insect words in the prime versus the target position in either the MEG or the fMRI session (no interactions between Group and Position, Fs $<2.15$; ps $>0.15)$

\footnotetext{
${ }^{2}$ In the fMRI study, a technical error meant that we failed to save the behavioral data of one control and three people with schizophrenia and so accuracy scores reflect the data of the remaining 19 control participants and 13 people with schizophrenia. We included all participants in the fMRI analysis as our scanning logs indicated that these participants responded consistently, and their MEG behavioral data suggested that they performed at the same level of accuracy as the rest the group.
}

\section{2. $M E G$ results}

\subsubsection{Indirect priming effect}

Within the $300-500 \mathrm{~ms}$ time window of interest, collapsed across temporal sites, the cluster-based permutation test revealed a significant difference between the schizophrenia and control groups in the indirect priming effect (a cluster from 428 to $453 \mathrm{~ms}$, although note that this is likely to underestimate the true extent of the effect, see http://www. fieldtriptoolbox.org/faq/). ${ }^{3}$ Planned follow-ups confirmed a significant indirect priming effect in the schizophrenia group (cluster-level $\mathrm{p}=0.0344$; cluster between 431 and $461 \mathrm{~ms}$ ), but no such effect in the control group. These data are shown in Fig. 4. The topographic map in Fig. 4 also confirms that the indirect priming effect in the schizophrenia group was centered over temporal sensors where previous MEG studies report maximal N400 modulation (e.g. Halgren et al., 2002; Helenius et al., 1998; Lau et al., 2013).

\subsubsection{Direct priming effect}

In contrast, the mass univariate analysis failed to show any difference between the two groups in the direct priming effect within the N400 time window. In addition, a mass univariate analyses that collapsed across the two groups also failed to show evidence of a significant direct priming effect within the N400 time window.

In Supplementary Fig. 1, we show the event-related field responses evoked by targets in all three conditions - directly related, indirectly related and unrelated target words - over frontal, parietal, temporal, and occipital sensors, in both groups.

\subsection{FMRI results}

\subsubsection{Temporal fusiform cortex}

3.3.1.1. Indirect priming effect. In comparing the unrelated vs. indirectly related word-pairs, the SPM revealed evidence of a Group by Relatedness interaction within the left fusiform cortex (small volume corrected voxel-level $\mathrm{p}_{\mathrm{FWE}}$ 0.0188; $\mathrm{Z}=3.83$; peak coordinates: -22 $-32-24)$, see Fig. 5. ${ }^{4}$ Follow-ups showed that this interaction was driven by a significant indirect priming effect (less hemodynamic activity to the indirectly related word-pairs than to the unrelated word-pairs) in the schizophrenia group, $\mathrm{t}(15)=-4.764, \mathrm{p}<0.001$, but no such effect in the control group, $\mathrm{t}(19)=1.626, \mathrm{p}=0.12$.

3.3.1.2. Direct priming effect. In contrast, there was no evidence of a Group by Relatedness interaction in contrasting the directly related and unrelated word-pairs; that is, the two groups failed to show any significant difference in the direct semantic priming effect within this region.

We also carried out a post-hoc full $3 \times 2$ ANOVA that included all three conditions. This revealed a significant effect within the left fusiform with the same peak coordinates $(-22-32-24)$ as for our planned $2 \times 2$ ANOVA that contrasted the indirectly related and unrelated word-pairs. In Supplementary materials, we show contrast estimates within this region in all three conditions in both groups (see Supplementary Fig. 2).

\footnotetext{
${ }^{3}$ We also carried out an ANCOVA that compared the two groups on the difference waveform (unrelated minus indirectly related), averaged across all time points within this temporal cluster, while covarying for pre-morbid verbal IQ, as assessed using the NAART (Blair and Spreen, 1989). The between-group difference remained significant, $\mathrm{F}(1,33)=8.823, \mathrm{p}<0.01$.

${ }^{4}$ We also carried out an ANCOVA (dependent variable: average activity across all voxels within the ROI, which were activated at the $p<0.005$ whole brain voxel-wise threshold) in which we covaried for pre-morbid verbal IQ, as assessed using the NAART (Blair and Spreen, 1989). The Group by Relatedness effect remained significant, $\mathrm{F}(1,33)=14.932$, $\mathrm{p}<0.001$
} 

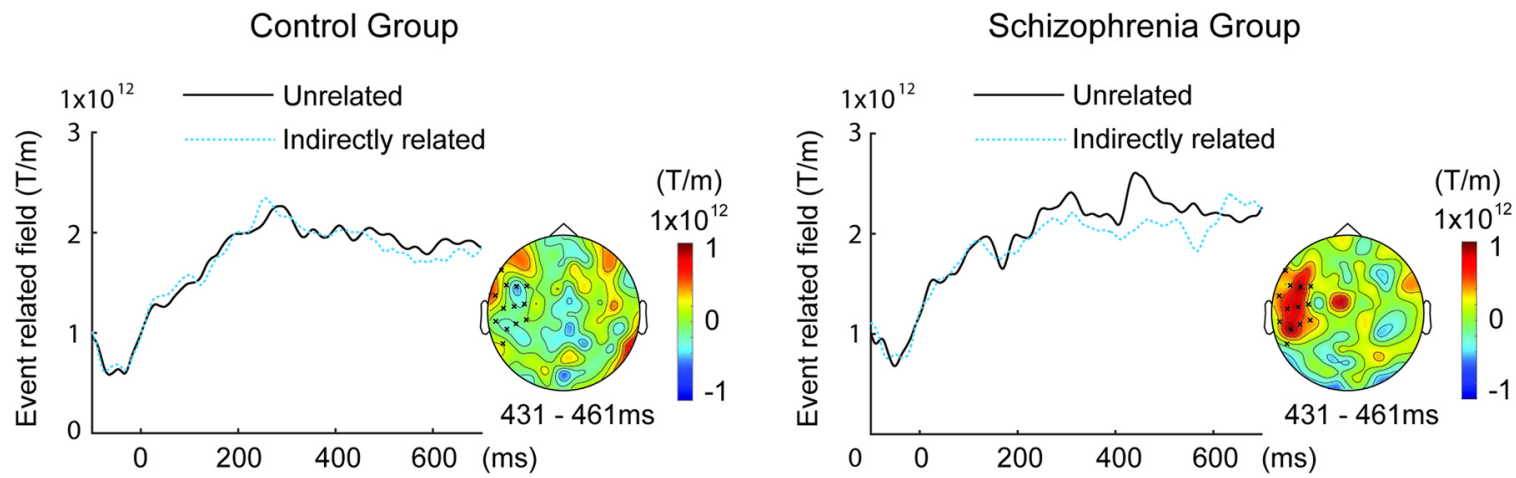

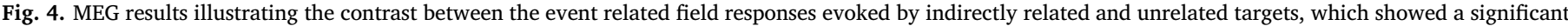

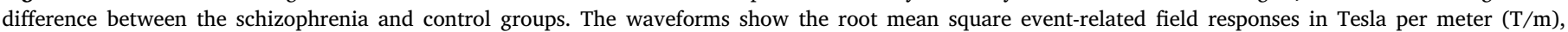

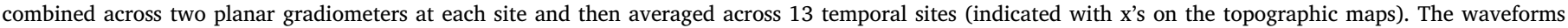

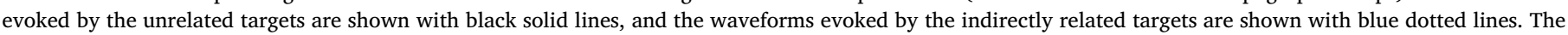

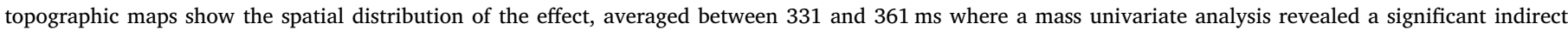

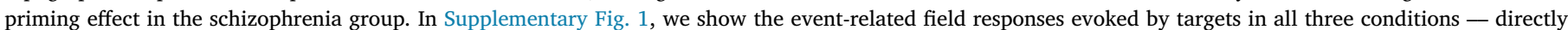

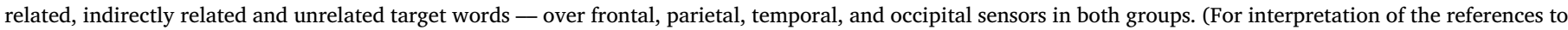
color in this figure legend, the reader is referred to the web version of this article.)
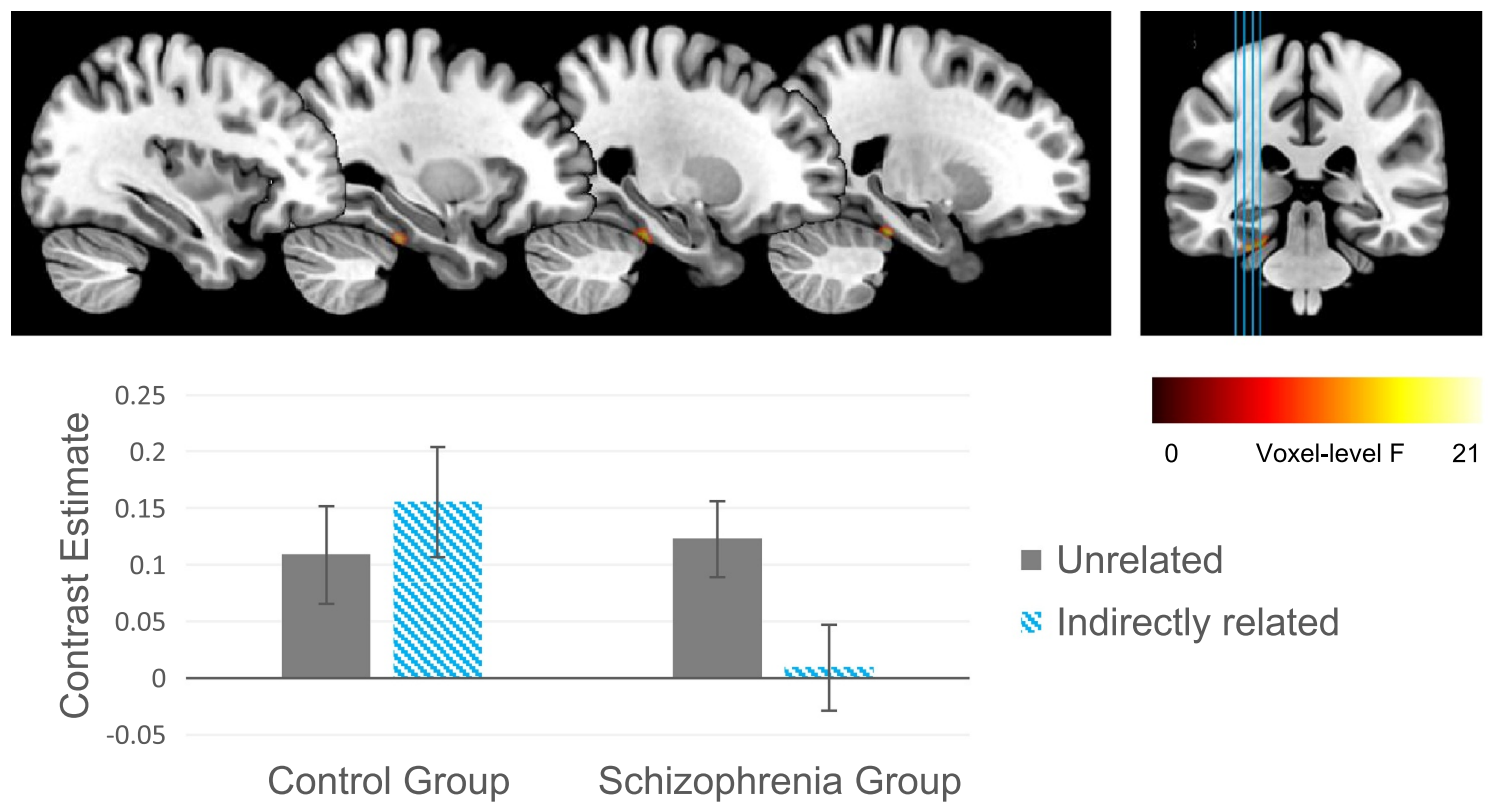

\section{- Unrelated \\ $\mathrm{s}$ Indirectly related}

\section{Control Group Schizophrenia Group}

Fig. 5. fMRI results illustrating voxels that showed a significant difference in the automatic indirect priming effect between the schizophrenia and control groups. Top row: The Group (control vs. schizophrenia) $\times$ Relatedness (indirectly related vs. unrelated) interaction map revealed a significant hemodynamic effect within the left temporal fusiform cortex (small volume corrected over right and left fusiform cortices voxel-level $\mathrm{p}_{\mathrm{FWE}}=0.018$ ). $\mathrm{F}$ values are plotted (see color bar for scale). Left: Sagittal slices at $\mathrm{x}=[-35-30-26-22]$. Right: Coronal slice at $\mathrm{y}=-33$, with blue vertical lines indicating the position of the sagittal slices. Bottom row: Bar graphs display the contrast estimate, averaged across all voxels that were activated within the left fusiform cortex (at the $\mathrm{p}<0.005$ whole brain voxel-wise threshold) to unrelated word-pairs (gray, solid) and indirectly related word-pairs (blue, shaded), within the control and schizophrenia group. Error bars reflect standard error. In Supplementary Fig. 2, we show the contrast estimates within this region to all three conditions — directly related, indirectly related and unrelated - in both groups. (For interpretation of the references to color in this figure legend, the reader is referred to the web version of this article.)

\subsubsection{Left anterior superior/middle temporal cortex}

Within the left lateral anterior temporal region of interest, we saw no evidence of a Group by Relatedness interaction in contrasting unrelated word-pairs with either directly related or with indirectly related word-pairs. We also failed to see a main effect of Relatedness in either of these analyses. Because we had previously observed a direct priming effect within the left lateral anterior temporal region in a group of young healthy controls (Lau et al., 2013), we carried out a post-hoc analysis in the control group that contrasted directly related and unrelated word-pairs within this region. This failed to show a significant direct priming effect, although modulation was seen at an uncorrected voxel-wise threshold of $\mathrm{p}<0.05$.

Finally, to check whether there was evidence of a significant direct or indirect hemodynamic priming effect in either group outside our regions of interest, we carried out exploratory whole-brain analyses within each group. In the schizophrenia group, we observed only a single large cluster that showed significantly less activity to the indirectly related than the unrelated word-pairs $(k=19,614$; clusterlevel $\mathrm{p}_{\mathrm{FWE}}<0.0005$, across the whole brain). This cluster included the voxels within the left fusiform region (see above), as well as within the hippocampus, parahippocampal gyrus, medial parietal cortex, medial and ventral occipital cortex and cerebellum (see Table 3). We saw no other regions modulated by indirect priming or direct priming in the schizophrenia group, even at this liberal statistical threshold. We also saw no significant clusters in the control group.

Finally, the comparison of all word-pairs and the implicit baseline 
revealed widespread activity in both the schizophrenia and the control group (see Fig. 6, Table 4), with no significant differences between the two groups.

\section{Discussion}

We used fMRI and MEG together with an automatic indirect semantic priming paradigm to spatiotemporally localize the automatic indirect semantic priming effect in schizophrenia. Results from both techniques provided clear neural evidence for a larger automatic indirect priming effect in people with schizophrenia than in demographically matched control participants: in the schizophrenia group, neural activity was reduced to target words that were preceded by indirectly related (versus unrelated) prime words, but no such effect was seen in the control group. Our MEG data temporally localized this effect to the N400 time window, while our fMRI data spatially localized the effect to the left temporal fusiform cortex. In contrast, we found no evidence of a larger direct priming effect in the schizophrenia relative to the control group.

Several previous studies have used ERP and/or fMRI methods separately to investigate the neural correlates of direct and indirect semantic priming in schizophrenia (ERP: e.g. Kreher et al., 2009, 2008; Kiang et al., 2008; fMRI: e.g. Kuperberg et al., 2007; Han et al., 2007; Wilson et al., 2013). The present study is novel in two main ways. First, a subset of participants took part in both MEG and fMRI sessions in which they viewed the same stimuli (counterbalanced over sessions) under the same experimental conditions and with the same task. This is important because it has been difficult to compare findings across previous studies that appear to show discrepant results, as a result of the different temporal and spatial resolutions of MEG/ERP and fMRI, the sensitivity of the semantic priming effect to precise experimental conditions and task demands, and the heterogeneity of schizophrenia. Second, unlike previous studies that have examined indirect priming in schizophrenia under semi-automatic conditions, we set up our experimental conditions to be highly automatic, eliminating top-down mechanisms of priming altogether (masking the prime, using an SOA of only $140 \mathrm{~ms}$, and employing a task that didn't require participants to identify the semantic relationship between the prime and target).

Our MEG and fMRI results were strikingly convergent in showing the same pattern of findings across the three conditions and the two groups. Our MEG finding of a larger automatic indirect priming effect in schizophrenia within the N400 time window replicates and extends our previous ERP work, which used similar stimuli and a similar task, but was carried out under less automatic experimental conditions (an SOA

Table 3

Results of an exploratory whole brain analysis within the schizophrenia group contrasting unrelated word-pairs with indirectly related word-pairs.

\begin{tabular}{lllll}
\hline Region & R/L & p-value & z-score & MNI (x, y, z) \\
\hline Fusiform cortex & L & $<0.0001$ & 4.05 & {$[-30 ;-30 ;-28]$} \\
& R & 0.0002 & 3.56 & {$[26 ;-38 ;-24]$} \\
Hippocampus & $\mathrm{R}$ & 0.0001 & 3.64 & {$[32 ;-12 ;-18]$} \\
Parahippocampal gyrus & $\mathrm{R}$ & 0.0004 & 3.34 & {$[28 ;-40 ;-2]$} \\
& $\mathrm{L}$ & 0.0008 & 3.15 & {$[-18 ;-36 ;-10]$} \\
Posterior cingulate cortex & $\mathrm{L}$ & 0.0006 & 3.23 & {$[-10 ;-48 ; 28]$} \\
Precuneus & $\mathrm{R}$ & 0.0007 & 3.21 & {$[18 ;-52 ; 16]$} \\
Occipital cortex (Lingual) & $\mathrm{R}$ & 0.0008 & 3.17 & {$[20 ;-82 ;-10]$} \\
Occipital cortex (Calcarine) & $\mathrm{L}$ & 0.001 & 3.02 & {$[-12 ;-54 ; 8]$} \\
Cerebellum & $\mathrm{R}$ & 0.0008 & 3.16 & {$[18 ;-42 ;-20]$} \\
\hline
\end{tabular}

A single cluster showed significantly more hemodynamic activity to unrelated word pairs than indirectly related word-pairs in the schizophrenia group (number of contiguous voxels: 19,614; cluster-level p value, FWE corrected: $\mathrm{p}<0.0005$ ). Anatomical locations and MNI coordinates correspond to the $\mathrm{p}$ values and z-scores of representative peaks within each cluster. We used the AAL atlas to identify the anatomical regions reported. Only one peak per anatomical region is reported for each hemisphere.

\section{Word-pairs > Implicit baseline}
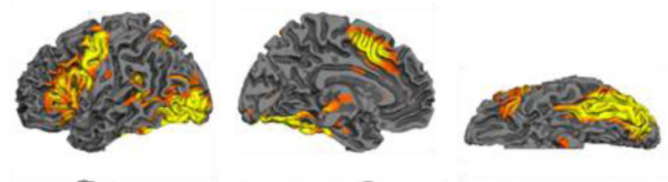

$\mathrm{L}$
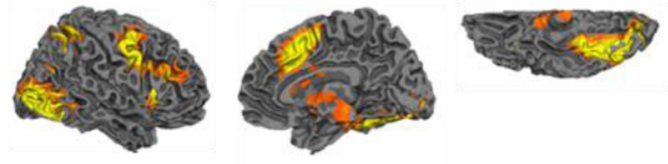

Implicit baseline > Word-pairs
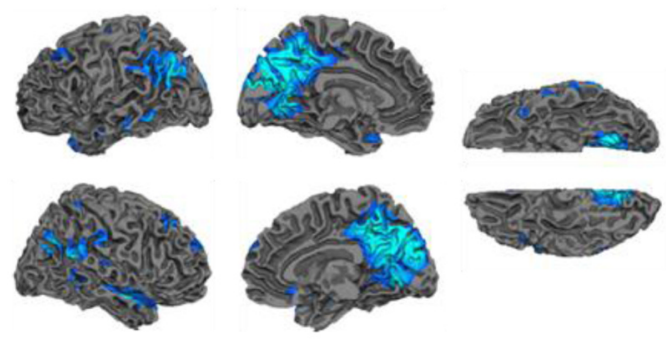

$\mathrm{L}$

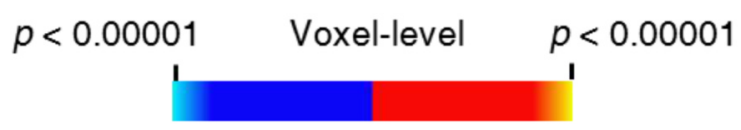

Fig. 6. Statistical maps showing the contrast of all word-pairs versus the implicit baseline, collapsed across the schizophrenia and control groups (no differences were found between the two groups). Effects are shown at a voxel-level significance threshold of $\mathrm{p}<0.005$ and include clusters consisting of 10 or more contiguous voxels. Top: Yellow-red: more activity to word-pairs than the implicit baseline was seen within a bilateral but left lateralized network distributed across the frontal cortices (left inferior frontal cortices and left and right precentral cortices), temporal cortices (the temporal fusiform cortices and, on the left, the middle temporal cortex), occipital cortices, right cingulate cortex, as well as subcortical regions (left and right putamen and pallidum extending into the thalamus, data not shown). Bottom: Blue: less activity to word-pairs than the implicit baseline was observed bilaterally within the occipital cortices, extending into the precuneus and post-central cortices, posterior regions of the temporal cortices, and the inferior parietal lobule. (For interpretation of the references to color in this figure legend, the reader is referred to the web version of this article.)

of $350 \mathrm{~ms}$ and no masking of the prime: the implicit task in Kreher et al., 2009). Our fMRI finding shows, for the first time, evidence of an enhanced automatic indirect priming effect in schizophrenia in the hemodynamic response (as discussed in the Introduction, previous fMRI studies, carried out under less automatic conditions, have reported reverse hemodynamic priming effects in schizophrenia, see Han et al., 2007; Kuperberg et al., 2007; Wilson et al., 2013). The fact that the enhanced indirect priming effect in schizophrenia localized to the temporal cortex, in the absence of prefrontal activity, suggests that we successfully eliminated top-down mechanisms and isolated the effects of highly automatic bottom-up processes (see Dehaene et al., 2001).

In the control group, neither MEG nor fMRI detected evidence of either an indirect or a direct priming effect. The absence of an automatic indirect priming effect in the controls was not surprising: as noted in the Introduction, indirect semantic priming is minimal or absent in healthy adults (de Groot, 1983; McNamara and Altarriba, 1988). The absence of an automatic direct priming effect in the control group was, however, more surprising because we had previously shown such an effect in both fMRI and MEG using this paradigm in a group of young healthy adults (Lau et al., 2013). One possible reason for this discrepancy is that the healthy adults in the present study were, on 
Table 4

Hemodynamic modulation contrasting all word pairs with the implicit baseline (collapsed across the control and schizophrenia groups).

\begin{tabular}{|c|c|c|c|c|c|}
\hline Region & $\mathbf{R} / \mathbf{L}$ & Peak voxel p-value & z-score & $\operatorname{MNI}(x, y, z)$ & Cluster level p-value \\
\hline \multicolumn{6}{|l|}{ A. Word pairs > Implicit baseline } \\
\hline Inferior parietal lobule (other) & $\mathrm{L}$ & $<0.0001$ & 5.62 & $-32,-54,50$ & $p_{\mathrm{FWE}}<0.0001 k=13,158$ \\
\hline Superior temporal cortex (posterior) & $\mathrm{L}$ & $<0.0001$ & 4.95 & $-46,-42,18$ & \\
\hline Fusiform cortex (temporal) & $\mathrm{L}$ & $<0.0001$ & $\infty$ & $-36,-44,-20$ & \\
\hline Fusiform cortex (occipital) & $\mathrm{L}$ & $<0.0001$ & 7.57 & $-38,-68,-14$ & \\
\hline Occipital cortex (lingual) & $\mathrm{L}$ & $<0.0001$ & 6.52 & $-24,-90,-12$ & \\
\hline Occipital cortex (lateral) & $\mathrm{L}$ & $<0.0001$ & 6.49 & $-20,-92,-10$ & \\
\hline \multirow[t]{2}{*}{ Cerebellum } & $\mathrm{L}$ & $<0.0001$ & 5.73 & $-6,-72,-22$ & \\
\hline & $\mathrm{R}$ & $<0.0001$ & 4.84 & $8,-66,-22$ & \\
\hline Inferior frontal gyrus (pars opercularis) & $\mathrm{L}$ & $<0.0001$ & 4.20 & $-48,10,8$ & $p_{\mathrm{FWE}}<0.0001 k=25,069$ \\
\hline Inferior frontal gyrus (pars triangularis) & $\mathrm{L}$ & $<0.0001$ & 5.44 & $-40,26,12$ & \\
\hline Inferior frontal gyrus (pars orbitalis) & $\mathrm{L}$ & $<0.0001$ & 3.86 & $-38,28,-16$ & \\
\hline Middle frontal cortex & $\mathrm{R}$ & $<0.0001$ & 4.74 & $30,28,16$ & \\
\hline \multirow[t]{2}{*}{ Precentral gyrus } & $\mathrm{L}$ & $<0.0001$ & 6.82 & $-46,-2,60$ & \\
\hline & $\mathrm{R}$ & $<0.0001$ & 5.79 & $56,-4,50$ & \\
\hline Supplementary motor area & $\mathrm{L}$ & $<0.0001$ & 6.60 & $0,8,54$ & \\
\hline \multirow[t]{2}{*}{ Insula } & $\mathrm{L}$ & $<0.0001$ & 5.30 & $-26,24,4$ & \\
\hline & $\mathrm{R}$ & $<0.0001$ & 4.66 & $30,24,4$ & \\
\hline Postcentral gyrus & $\mathrm{L}$ & $<0.0001$ & 6.77 & $-56,-4,50$ & \\
\hline Middle cingulate cortex & $\mathrm{R}$ & $<0.0001$ & 5.91 & $8,20,40$ & \\
\hline \multirow[t]{2}{*}{ Basal ganglia (pallidum) } & $\mathrm{L}$ & $<0.0001$ & 4.63 & $-22,-8,8$ & \\
\hline & $\mathrm{R}$ & $<0.0001$ & 4.13 & $24,-8,6$ & \\
\hline \multirow[t]{2}{*}{ Basal ganglia (putamen) } & $\mathrm{L}$ & $<0.0001$ & 4.50 & $-24,10,-2$ & \\
\hline & $\mathrm{R}$ & $<0.0001$ & 4.63 & $26,6,-2$ & \\
\hline Thalamus & $\mathrm{R}$ & $<0.0001$ & 4.57 & $12,-10,0$ & \\
\hline Inferior parietal lobule (other) & $\mathrm{R}$ & $<0.0001$ & 5.91 & $34,-52,48$ & $p_{\mathrm{FWE}}<0.0001 k=7,661$ \\
\hline Fusiform cortex (occipital) & $\mathrm{R}$ & $<0.0001$ & 6.12 & $38,-48,-22$ & \\
\hline Occipital cortex (lateral) & $\mathrm{R}$ & $<0.0001$ & 6.45 & $38,-86,0$ & \\
\hline
\end{tabular}

B. Word pairs < Implicit baseline

\begin{tabular}{|c|c|c|c|c|c|}
\hline \multirow[t]{2}{*}{ Postcentral gyrus } & $\mathrm{L}$ & $<0.0001$ & 3.95 & $-26,-42,62$ & \multirow[t]{2}{*}{$p_{\mathrm{FWE}}<0.0001 k=13,014$} \\
\hline & $\mathrm{R}$ & 0.0016 & 2.94 & $24,-42,62$ & \\
\hline Middle cingulate cortex & $\mathrm{L}$ & 0.0006 & 3.26 & $0,-14,46$ & \\
\hline \multirow[t]{2}{*}{ Precuneus } & $\mathrm{L}$ & $<0.0001$ & 6.14 & $-8,-48,46$ & \\
\hline & $\mathrm{R}$ & $<0.0001$ & 5.06 & $8,-54,32$ & \\
\hline \multirow[t]{2}{*}{ Occipital cortex (cuneus) } & $\mathrm{L}$ & $<0.0001$ & 6.01 & $-8,-90,20$ & \\
\hline & $\mathrm{R}$ & $<0.0001$ & 5.84 & $6,-70,30$ & \\
\hline Occipital cortex (calcarine) & $\mathrm{L}$ & $<0.0001$ & 4.73 & $-8,-62,10$ & \\
\hline \multirow[t]{2}{*}{ Occipital cortex (lingual) } & $\mathrm{L}$ & $<0.0001$ & 4.92 & $-6,-70,-2$ & \\
\hline & $\mathrm{R}$ & $<0.0001$ & 4.61 & $10,-70,0$ & \\
\hline Inferior parietal lobule (supramarginal gyrus) & $\mathrm{R}$ & $<0.0001$ & 3.79 & $60,-26,34$ & \multirow[t]{4}{*}{$p_{\mathrm{FWE}}<0.0080 k=1,789$} \\
\hline Superior temporal cortex (posterior) & $\mathrm{R}$ & $<0.0001$ & 4.16 & $62,-58,22$ & \\
\hline Middle temporal cortex (posterior) & $\mathrm{R}$ & $<0.0001$ & 3.96 & $64,-52,0$ & \\
\hline Occipital cortex (lateral) & $\mathrm{R}$ & $<0.0001$ & 4.90 & $48,-76,30$ & \\
\hline Inferior parietal lobule (angular gyrus) & $\mathrm{L}$ & $<0.0001$ & 4.55 & $-60,-64,28$ & \multirow[t]{4}{*}{$p_{\mathrm{FWE}}<0.0050 k=1,971$} \\
\hline Inferior parietal lobule (supramarginal gyrus) & $\mathrm{L}$ & $<0.0001$ & 3.99 & $-64,-50,36$ & \\
\hline Middle temporal cortex (posterior) & $\mathrm{L}$ & $<0.0001$ & 4.49 & $-62,-62,24$ & \\
\hline Occipital cortex (lateral) & $\mathrm{L}$ & $<0.0001$ & 4.82 & $-42,-84,34$ & \\
\hline
\end{tabular}

A. Regions showing more activity to the word-pairs than the implicit baseline, across both groups.

B. Regions showing less activity to the word-pairs than the implicit baseline, across both groups.

There were no regions that showed a significant difference between the two groups.

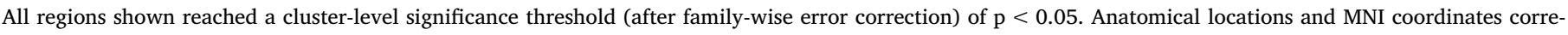

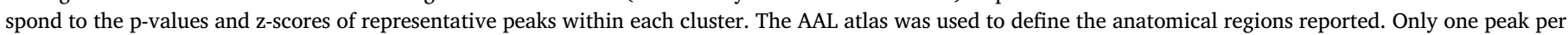

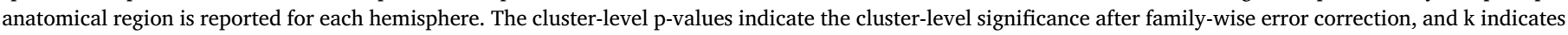
the number of contiguous voxels within each cluster.

average, over 20 years older than those who participated in our previous study. We suggest that the masked prime may have been less effective in initiating automatic semantic activity in these older adults: under these highly automatic conditions, there may not have been enough time for the activity from the masked prime to pre-active features at the semantic level. This of course limits any interpretation of the absence of an automatic direct priming effect at the semantic level in the schizophrenia group. On the other hand, the fact that we were still able to detect a significant automatic indirect priming effect in the same patients, using both techniques, gives us some clues about the mechanism of enhanced automatic indirect priming in schizophrenia. We turn to this question next.

\subsection{Mechanism of the enhanced indirect automatic priming effect in schizophrenia}

As outlined in the Introduction, an enhanced automatic indirect priming effect in schizophrenia could, in theory, result either from an unconstrained (or disinhibited) spread of activity across semantic memory (e.g. Manschreck et al., 1988), or from noisier lexical representations - that is, looser (less finely-tuned) mappings/connections between representations of word-form and semantic features (Brown and Kuperberg, 2015; Kuperberg et al., 2018a), see Fig. 1. The present set of findings cannot be easily explained by the former account; they are more consistent with the latter theory. This is for two reasons. 


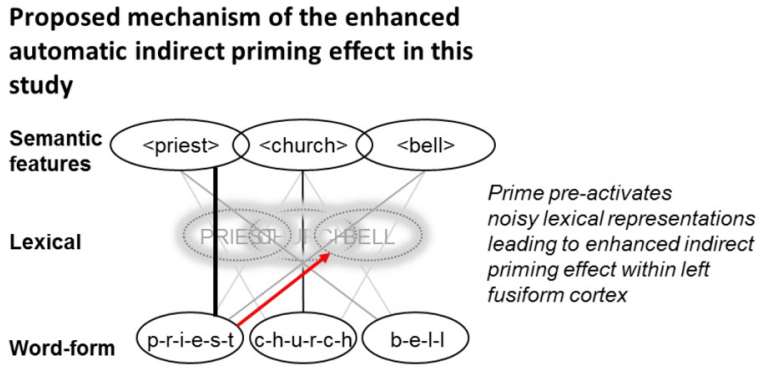

Fig. 7. Diagrammatic illustration (highly simplified) of proposed mechanism underlying the enhanced automatic indirect priming effect in schizophrenia in the present study. The connections between word-form and meaning are weaker (depicted as fainter lines) and less finely-tuned (no 1:1 mappings) than in healthy adults - that is, lexical representations are noisier. This may arise as a long-term consequence of adapting to sustained abnormal prediction error at the level of word-form and semantic features in schizophrenia. Here, the lexical representation, BELL, encodes mappings between $<$ bell $>$ and both b-e-l-l and p-r-i-e-s-t. Thus, after encountering the prime word, "priest", there is a direct route, depicted here with a red arrow, through which bottom-up activity can flow from its orthographic form (p-r-i-e-s-t) to pre-activate its indirectly related lexical representation (BELL). This means that if the indirectly related target word, "bell", appears very quickly after the prime, its processing will be facilitated at the lexical level, leading to an indirect priming effect within the left fusiform cortex. Under these highly automatic experimental conditions, in this older population, there was not enough time for the activity from the masked prime to reach all the way up to pre-activate features at the semantic level of representation. (For interpretation of the references to color in this figure legend, the reader is referred to the web version of this article.)

First, the 'unconstrained spread of semantic activity' account predicts that a larger indirect semantic priming effect in schizophrenia should necessarily be accompanied by a larger direct priming effect. This is because, according to this theory, in order to pre-activate a prime's indirectly related semantic features, it is first necessary to preactivate its directly related semantic features. In contrast, the 'noisy lexical representations' account can explain how an enhanced indirect priming effect can occur in schizophrenia without necessarily being accompanied by an enhanced direct priming effect. This is because it provides a direct pathway through which bottom-up automatic activity can flow from a prime's word-form representation to pre-activate its indirectly related representations, without this being mediated by the pre-activation of the prime's directly related representations.

Second, and relatedly, the 'unconstrained spread of semantic activity' account predicts that the enhanced indirect priming effect in schizophrenia should occur at the level of semantic features that were pre-activated by spreading activation from the prime. However, as discussed above (in relation to why we saw no direct automatic priming effect in the control group), in these older adult participants, under these highly automatic experimental conditions, there may not have been enough time for activity from the masked prime to reach all the way up to preactivate features at the semantic level. The 'noisy lexical representation' account, however, predicts that the indirect semantic priming effect in schizophrenia can occur at the lexical level of representation. This is because, according to this theory, the lexical representation, BELL, encodes not only mappings between the word-form, b-e-l-1, and the semantic features, $<$ bell $>$, but also between the word-form, p-r-i-e-s-t, and the semantic features, $<$ bell $>$. It is therefore possible for automatic bottom-up activity to flow from the prime, p-r-i-e-s-t, to pre-activate the lexical representation, BELL, even if this flow of activity doesn't reach the level of semantic features. Consistent with this idea, our fMRI findings show that the automatic indirect semantic effect in schizophrenia localized to the left fusiform cortex - a region that has been implicated in lexical processing at the interface between orthographic form and semantic features (Price and Devlin, 2011), and that is distinct from the anterior lateral temporal cortex that is associated with processing at the semantic level (Lambon Ralph and Patterson, 2008; Lambon Ralph et al., 2017; Lau et al., 2013). Note that, as outlined in the Introduction and in Fig. 1, the 'noisy lexical representations' account predicts that the automatic indirect semantic priming effect in schizophrenia can also occur at the level of semantic features. However, we would not have been able to detect this in this older population. In Fig. 7, we illustrate the proposed mechanism of the enhanced indirect priming effect in the schizophrenia group in the present study.

\subsection{Open questions}

If schizophrenia is indeed characterized by looser lexical mappings between word-form and meaning, this raises the question of how such abnormal connections might come about. One theory is that they arise as a long-term consequence of an abnormally large semantic prediction error in response to incoming stimuli in schizophrenia (for general discussion, see Fletcher and Frith, 2009; for discussion in relation to language processing, see Brown and Kuperberg, 2015). During normal language comprehension, healthy adults are thought to generate precise top-down predictions both at the level of semantic features and word-form, particularly in semantically constraining contexts. This means that incoming words that fulfill such predictions (e.g. the word, "priest", following the context, "The man went to confession and confessed his sins to the... ") will not produce a prediction error, either the level of word-form or semantic features (see Kuperberg and Jaeger, 2016). In schizophrenia, however, top-down predictions during language comprehension tend to be less precise and more dependent on general semantic relationships between individual words, rather than precise discourse representations of context (e.g. Kuperberg et al., 2006; Ditman et al., 2011; Swaab et al., 2013). Thus, following the context above, people with schizophrenia may be more likely to weakly pre-activate general schema-related semantic features (e.g. $<$ church $>,<$ priest $>,<$ bell $>$ ), without pre-activating upcoming word-forms. Thus, in people with schizophrenia, new bottom-up predictable input ("priest") would evoke a relatively large prediction error at the level of the word-form, p-r-i-e-s-t (because this word-form prediction was not generated at all), a small prediction error over the semantic features corresponding to $<$ priest $>$ and $<$ church $>$ (because these features were partially predicted), and the largest prediction error over the semantic features corresponding to $<$ bell $>$ (because these predicted semantic features mismatch those associated with the bottom-up input). There is evidence that prediction error triggers learning/adaptation, both in the language domain (e.g. Dell and Chang, 2014) and in non-linguistic domains (e.g. Rescorla and Wagner, 1972). Thus, over the longer term, an abnormally large prediction error to p-r-i-e-s-t and $<$ bell $>$ in schizophrenia might lead to adaptation such that the word-form, p-r-i-e-s-t, develops new connections to the semantic features of its indirect associates $(<$ bell $>$ ), leading to noisier lexical representations, just as depicted in Figs. 1B and 7. In other words, abnormally increased prediction error in schizophrenia might interfere with the maintenance of stable, precise lexical connections between the meanings and forms of words, leading to broader connections. Indeed, we have argued that sustained, abnormally increased prediction error may lead to weaker, less precise mappings across at multiple levels of the linguistic hierarchy in schizophrenia (see Brown and Kuperberg, 2015 for a review, and for evidence of abnormal top-down predictive effects at other levels of linguistic representation in schizophrenia, see Rabagliati et al. (in press), and Kuperberg et al. (2018b)).

A second open question concerns the relationship between the abnormalities described here and clinical symptoms of disorganized speech (thought disorder) in schizophrenia. In this study, the schizophrenia group included patients without significant thought disorder. While this suggests that, at a neural level, abnormally broad automatic lexico-semantic activity is not specific to thought disorder, it is possible that it may be more severe in thought-disordered patients, as suggested by previous studies (e.g. Moritz et al., 2003; Spitzer et al., 1993; Kreher et al., 2008). It will be therefore important to test this hypothesis in future studies that include a larger number of schizophrenia 
participants with a larger range of thought disorder. ${ }^{5}$

Finally, it is important to acknowledge that, from this study alone, the precise relationship between our MEG and fMRI findings is unclear. Although we observed an enhanced automatic indirect priming effect in the schizophrenia group using both techniques, we cannot tell whether the source of the MEG effect within the N400 time window was, in fact, the left fusiform cortex that was modulated in the fMRI session. While our fMRI results are likely to have captured the indirect priming effect on target words (because the prime was fully counterbalanced across conditions and participants), fMRI does not have the necessary temporal resolution to tell us when exactly this modulation occurred in relation to the target's onset; in theory, it could have reflected activity within or after the N400 time window. One way of addressing this question is to carry out a source analysis of the MEG sensor-level indirect priming effect between 300 and $500 \mathrm{~ms}$ in schizophrenia. We did attempt to carry out such an analysis using distributed source modeling methods (Hämäläinen and Sarvas, 1989; Gramfort et al., 2014), but this did not reveal any significant effect in the fusiform (or any other) region within this time window. This is likely because of limited power, particularly given that the relatively inferior and medial anatomical location of the fusiform cortex makes it challenging to source localize effects within this region. It will therefore be important to replicate the MEG study with a larger number of participants to determine whether the temporal fusiform cortex is indeed the source of the abnormally increased indirect priming effect within the N400 time window in schizophrenia.

\subsection{Conclusions}

In conclusion, our use of two complementary neuroimaging modalities, MEG and fMRI, in the same participants using the same highly automatic masked priming paradigm, provides converging neural evidence for abnormally broad automatic lexico-semantic activity in schizophrenia. Our MEG findings suggest that this automatic facilitation was evident within the N400 time window - the critical stage of accessing meaning from the form of words, while our fMRI findings suggest that it was realized as reduced activity within the left temporal fusiform cortex, which is thought to mediate the process of mapping orthographic word-form on to meaning.

\section{Acknowledgments}

This work was funded by the National Institute of Mental Health (R01MH071635 to G.R.K.). We are also grateful to the Sidney R. Baer, Jr. Foundation for their support of undergraduate students including Sarah Armstrong, Ju Hyung Kim, Emily O'Carroll and Gianna Wilkie, who contributed to recruitment, data collection and other aspects of the project. We thank Donald Goff for his support in patient recruitment, Nao Matsuda for technical MEG support, Sheraz Khan and Lin Wang for their help with MEG analysis, and Lotte Schoot for her help with fMRI analysis. This research was carried out at the Athinoula A. Martinos Center for Biomedical Imaging at the Massachusetts General Hospital, using resources provided by the Center for Functional Neuroimaging Technologies, P41EB015896, a P41 Biotechnology Resource Grant supported by the National Institute of Biomedical Imaging and Bioengineering (NIBIB), National Institutes of Health. This work also

\footnotetext{
${ }^{5}$ In the present study, we did carry out exploratory analyses to examine this relationship. These analyses did not reveal a significant correlation between SAPS global thought disorder scores and either (a) the MEG indirect priming effect (difference score averaged across the 431-461 ms cluster that showed the significant effect in the schizophrenia group), $p=0.83$, or (b) the hemodynamic indirect priming effect (difference score averaged across the temporal fusiform cluster that showed the significant effect in the schizophrenia group), $\mathrm{p}=0.52$. However, we cannot infer the absence of a true correlation from this null result, particularly given the relatively small number of patients and the limited range of thought disorder.
}

involved the use of instrumentation supported by the NIH Shared Instrumentation Grant Program, specifically, grant numbers S10RR014978 and S10RR021110.

\section{Appendix A. Supporting information}

Supplementary data associated with this article can be found in the online version at doi:10.1016/j.neuropsychologia.2018.10.024.

\section{References}

Andreasen, N.C., 1984a. Scale for the Assessment of Negative Symptoms (SANS). The University of Iowa, Iowa City.

Andreasen, N.C., 1984b. Scale for the Assessment of Positive Symptoms (SAPS). The University of Iowa, Iowa City, IA.

Barch, D.M., Cohen, J.D., Servan-Schreiber, D., Steingard, S., Steinhauer, S., van Kammen, D., 1996. Semantic priming in schizophrenia: an examination of spreading activation using word pronunciation and multiple SOAs. J. Abnorm. Psychol. 105 (4), 592-601. https://doi.org/10.1037/0021-843x.105.4.592.

Blair, J.R., Spreen, O., 1989. Predicting premorbid IQ: a revision of the National Adult Reading Test. Clin. Neuropsychol. 3 (2), 129-136. https://doi.org/10.1080/ 13854048908403285.

Bleuler, E., 1911/1950. Dementia Praecox, or the Group of Schizophrenias (J. Zinker, Trans.). International Universities Press, New York.

Brett, M., Anton, J., Valabregue, R., Poline, J.B., 2002. Region of interest analysis using the MarsBar toolbox for SPM 99. Paper presented at the Neurolmage.

Brown, M., Kuperberg, G.R., 2015. A hierarchical generative framework of language processing: Linking language perception, interpretation, and production abnormalities in schizophrenia. Front. Hum. Neurosci. 9, 643. https://doi.org/10.3389/fnhum. 2015.00643.

Burock, M.A., Dale, A.M., 2000. Estimation and detection of event-related fMRI signals with temporally correlated noise: a statistically efficient and unbiased approach. Hum. Brain Mapp. 11 (4), 249-260.

Chapin, K., McCown, J., Vann, L., Kenney, D., Youssef, I., 1992. Activation and facilitation in the lexicon of schizophrenics. Schizophr. Res. 6, 251-255. https://doi.org/10 1016/0920-9964(92)90008-S.

Chwilla, D.J., Kolk, H.H., 2002. Three-step priming in lexical decision. Mem. Cogn. 30 (2), 217-225.

de Groot, A.M.B., 1983. The range of automatic spreading activation in word priming. J. Verbal Learn. Verbal Behav. 22, 417-436. https://doi.org/10.1016/S0022-5371(83) 90273-6.

Dehaene, S., Naccache, L., Cohen, L., Bihan, D.L., Mangin, J.F., Poline, J.B., Riviere, D., 2001. Cerebral mechanisms of word masking and unconscious repetition priming. Nat. Neurosci. 4 (7), 752-758. https://doi.org/10.1038/89551.

Dell, G.S., Chang, F., 2014. The P-chain: relating sentence production and its disorders to comprehension and acquisition. Philos. Trans. R. Soc. B: Biol. Sci. 369 (1634), 20120394. https://doi.org/10.1098/rstb.2012.0394.

Ditman, T., Goff, D., Kuperberg, G.R., 2011. Slow and steady: sustained effects of lexicosemantic associations can mediate referential impairments in schizophrenia. Cogn. Affect. Behav. Neurosci. 11 (2), 245-258. https://doi.org/10.3758/s13415-0110020-7.

Fletcher, P.C., Frith, C.D., 2009. Perceiving is believing: a Bayesian approach to explaining the positive symptoms of schizophrenia. Nat. Rev. Neurosci. 10 (1), 48-58.

Gardner, D.M., Murphy, A.L., O'Donnell, H., Centorrino, F., Baldessarini, R.J., 2010. International consensus study of antipsychotic dosing. Am. J. Psychiatry 167 (6), 686-693. https://doi.org/10.1176/appi.ajp.2009.09060802.

Gold, B.T., Balota, D.A., Jones, S.J., Powell, D.K., Smith, C.D., Andersen, A.H., 2006 Dissociation of automatic and strategic lexical-semantics: functional magnetic resonance imaging evidence for differing roles of multiple frontotemporal regions. J. Neurosci. 26 (24), 6523-6532. https://doi.org/10.1523/JNEUROSCI.0808-06.2006.

Gopal, S., Miller, R.L., Michael, A., Adali, T., Cetin, M., Rachakonda, S., Calhoun, V.D., 2016. Spatial variance in resting fMRI networks of schizophrenia patients: an independent vector analysis. Schizophr. Bull. 42 (1), 152-160. https://doi.org/10. 1093/schbul/sbv085.

Gramfort, A., Luessi, M., Larson, E., Engemann, D.A., Strohmeier, D., Brodbeck, C., Hämäläinen, M.S., 2014. MNE software for processing MEG and EEG data. NeuroImage 86, 446-460. https://doi.org/10.1016/j.neuroimage.2013.10.027.

Groppe, D.M., Urbach, T.P., Kutas, M., 2011. Mass univariate analysis of event-related brain potentials/fields I: a critical tutorial review. Psychophysiology 48 (12), 1711-1725. https://doi.org/10.1111/j.1469-8986.2011.01273.x.

Halgren, E., Dhond, R.P., Christensen, N., Van Petten, C., Marinkovic, K., Lewine, J.D., Dale, A.M., 2002. N400-like magnetoencephalography responses modulated by semantic context, word frequency, and lexical class in sentences. NeuroImage 17 (3), 1101-1116. https://doi.org/10.1006/nimg.2002.1268.

Hämäläinen, M.S., Sarvas, J., 1989. Realistic conductivity geometry model of the human head for interpretation of neuromagnetic data. IEEE Trans. Biomed. Eng. 36 (2), 165-171. https://doi.org/10.1109/10.16463.

Han, S.D., Nestor, P.G., Hale-Spencer, M., Cohen, A., Niznikiewicz, M., McCarley, R.W. Wible, C.G., 2007. Functional neuroimaging of word priming in males with chronic schizophrenia. NeuroImage 35 (1), 273-282. https://doi.org/10.1016/j.neuroimage. 2006.11.029.

Helenius, P., Salmelin, R., Service, E., Connolly, J., 1998. Distinct time courses of word 
and context comprehension in the left temporal cortex. Brain 121 (6), 1133-1142. https://doi.org/10.1093/brain/121.6.1133.

Hollingshead, A.B., 1965. Two Factor Index of Social Position. Yale University Press, New Haven, CT.

Kiang, M., Kutas, M., Light, G.A., Braff, D.L., 2008. An event-related brain potential study of direct and indirect semantic priming in schizophrenia. Am. J. Psychiatry 165 (1), 74-81. https://doi.org/10.1176/appi.ajp.2007.07050763.

Kiefer, M., Martens, U., Weisbrod, M., Hermle, L., Spitzer, M., 2009. Increased unconscious semantic activation in schizophrenia patients with formal thought disorder. Schizophr. Res. 114 (1-3), 79-83. https://doi.org/10.1016/j.schres.2009.07. 024.

Kreher, D.A., Goff, D., Kuperberg, G.R., 2009. Why all the confusion? Experimental task explains discrepant semantic priming effects in schizophrenia under "automatic" conditions: evidence from event-related potentials. Schizophr. Res. 111 (1-3), 174-181. https://doi.org/10.1016/j.schres.2009.03.013.

Kreher, D.A., Holcomb, P.J., Goff, D., Kuperberg, G.R., 2008. Neural evidence for faster and further automatic spreading activation in schizophrenic thought disorder. Schizophr. Bull. 34 (3), 473-482. https://doi.org/10.1093/schbul/sbm108.

Kreher, D.A., Holcomb, P.J., Kuperberg, G.R., 2006. An electrophysiological investigation of indirect semantic priming. Psychophysiology 43 (6), 550-563. https://doi.org/10. 1111/j.1469-8986.2006.00460.x.

Kuperberg, G.R., Deckersbach, T., Holt, D.J., Goff, D., West, W.C., 2007. Increased temporal and prefrontal activity in response to semantic associations in schizophrenia. Arch. Gen. Psychiatry 64 (2), 138-151. https://doi.org/10.1001/archpsyc.64.2.138.

Kuperberg, G.R., Delaney-Busch, N., Fanucci, K., Blackford, T., 2018a. Priming Production: neural evidence for enhanced automatic semantic activity immediately preceding language production in schizophrenia. NeuroImage: Clin. 18, 74-85. https://doi.org/10.1016/j.nicl.2017.12.026.

Kuperberg, G.R., Ditman, T., Choi Perrachione, A., 2018b. When proactivity fails: an electrophysiological study of establishing reference in schizophrenia. Biol. Psychiatry Cogn. Neurosci. NeuroImaging 3 (1), 77-87. https://doi.org/10.1016/j.bpsc. 2017. 09.007.

Kuperberg, G.R., Jaeger, T.F., 2016. What do we mean by prediction in language comprehension? Lang. Cogn. Neurosci. 31 (1), 32-59. https://doi.org/10.1080/ 23273798.2015.1102299.

Kuperberg, G.R., Lakshmanan, B.M., Greve, D.N., West, W.C., 2008. Task and semantic relationship influence both the polarity and localization of hemodynamic modulation during lexico-semantic processing. Hum. Brain Mapp. 29 (5), 544-561. https://doi. org/10.1002/hbm.20419.

Kuperberg, G.R., Sitnikova, T., Goff, D., Holcomb, P.J., 2006. Making sense of sentences in schizophrenia: electrophysiological evidence for abnormal interactions between semantic and syntactic processing. J. Abnorm. Psychol. 115 (2), 251-265. https:// doi.org/10.1037/0021-843X.115.2.251.

Kutas, M., Federmeier, K.D., 2011. Thirty years and counting: finding meaning in the N400 component of the event-related brain potential (ERP). Annu. Rev. Psychol. 62, 621-647. https://doi.org/10.1146/annurev.psych.093008.131123.

Lambon Ralph, M.A., Jefferies, E., Patterson, K., Rogers, T.T., 2017. The neural and computational bases of semantic cognition. Nat. Rev. Neurosci. 18 (1), 42-55. https://doi.org/10.1038/nrn.2016.150.

Lambon Ralph, M.A., Patterson, K., 2008. Generalization and differentiation in semantic memory: insights from semantic dementia. Ann. N.Y. Acad. Sci. 1124, 61-76. https:// doi.org/10.1196/annals.1440.006.

Landauer, T.K., Dumais, S.T., 1997. A solution to Plato's problem: the latent semantic analysis theory of acquisition, induction, and representation of knowledge. Psychol. Rev. 104 (2), 211-240. https://doi.org/10.1037/0033-295x.104.2.211.

Landauer, T.K., Foltz, P.W., Laham, D., 1998. An introduction to Latent Semantic Analysis. Discourse Process 25 (2-3), 259-284. https://doi.org/10.1080/ 01638539809545028.

Lau, E.F., Gramfort, A., Hämäläinen, M.S., Kuperberg, G.R., 2013. Automatic semantic facilitation in anterior temporal cortex revealed through multimodal neuroimaging J. Neurosci. 33 (43), 17174-17181. https://doi.org/10.1523/Jneurosci.1018-13. 2013.

Manschreck, T.C., Maher, B.A., Milavetz, J.J., Ames, D., Weisstein, C.C., Schneyer, M.L., 1988. Semantic priming in thought disordered schizophrenic patients. Schizophr. Res. 1, 61-66. https://doi.org/10.1016/0920-9964(88)90041-2.

Marcel, A.J., 1983. Conscious and unconscious perception: an approach to the relations between phenomenal experience and perceptual processes. Cogn. Psychol. 15 (2), 238-300. https://doi.org/10.1016/0010-0285(83)90010-5.

Maris, E., Oostenveld, R., 2007. Nonparametric statistical testing of EEG- and MEG-data. J. Neurosci. Methods 164 (1), 177-190. https://doi.org/10.1016/j.jneumeth.2007. 03.024 .

Mathalon, D.H., Faustman, W.O., Ford, J.M., 2002. N400 and automatic semantic processing abnormalities in patients with schizophrenia. Arch. Gen. Psychiatry 59 (7), 641-648. https://doi.org/10.1001/archpsyc.59.7.641.

Mazaika, P.K., Hoeft, F., Glover, G.H., Reiss, A.L., 2009. ArtRepair: Center for Interdisciplinary Brain Sciences Research. Stanford University, Stanford, CA.

McNamara, T.P., Altarriba, J., 1988. Depth of spreading activation revisited: semantic mediated priming occurs in lexical decisions. J. Mem. Lang. 27 (5), 545-559. https:// doi.org/10.1016/0749-596x(88)90025-3.

Mikl, M., Marecek, R., Hlustik, P., Pavlicova, M., Drastich, A., Chlebus, P., Krupa, P., 2008. Effects of spatial smoothing on fMRI group inferences. Magn. Reson. Imaging 26 (4), 490-503. https://doi.org/10.1016/j.mri.2007.08.006.

Misra, M., Holcomb, P.J., 2003. Event-related potential indices of masked repetition priming. Psychophysiology 40 (1), 115-130. https://doi.org/10.1111/1469-8986. 00012.

Moritz, S., Woodward, T.S., Kuppers, D., Lausen, A., Schickel, M., 2003. Increased automatic spreading of activation in thought-disordered schizophrenic patients. Schizophr. Res. 59 (2-3), 181-186. https://doi.org/10.1016/S0920-9964(01) 00337-1.

Mozes, S., Whitfield-Gabrieli, S., 2011. Artifact Detection Toolbox (ART). MIT: GabrieliLaboratory, Cambridge, MA.

Neely, J.H., 1991. Semantic priming effects in visual word recognition: a selective review of current findings and theories. In: Besner, D., Humphreys, G.W. (Eds.), Basic Processes in Reading and Visual Word Recognition. Erlbaum, Hillsdale, NJ, pp. 264-333.

Ober, B.A., Vinogradov, S., Shenaut, G.K., 1995. Semantic priming of category relations in schizophrenia. Neuropsychology 9 (2), 220-228. https://doi.org/10.1037//08944105.9.2.220.

Oldfield, R.C., 1971. The assessment and analysis of handedness: the Edinburgh inventory. Neuropsychologia 9 (1), 97-113. https://doi.org/10.1016/0028-3932(71) 90067-4.

Oostenveld, R., Fries, P., Maris, E., Schoffelen, J.-M., 2011. FieldTrip: open source software for advanced analysis of MEG, EEG, and invasive electrophysiological data. Comput. Intell. Neurosci. 2011, 1. https://doi.org/10.1155/2011/156869.

Price, C.J., Devlin, J.T., 2011. The Interactive Account of ventral occipitotemporal contributions to reading. Trends Cogn. Sci. 15 (6), 246-253. https://doi.org/10.1016/J. Tics.2011.04.001.

Rabagliati, H., Delaney-Busch, N., Busch, J., Snedeker, J., Kuperberg, G.R., 2018. Spared bottom-up but impaired top-down interactive effects during naturalistic language processing in schizophrenia: Evidence from the visual world paradigm. Psychol. Med. 1-11. https://doi.org/10.1017/S0033291718001952.

Rademacher, J., Galaburda, A.M., Kennedy, D.N., Filipek, P.A., Caviness, V.S., 1992. Human cerebral cortex localization, parcellation, and morphometry with magnetic resonance imaging. J. Cogn. Neurosci. 4 (4), 352-373. https://doi.org/10.1162/jocn. 1992.4.4.352.

Rescorla, R.A., Wagner, A.R., 1972. A theory of Pavlovian conditioning: variations in the effectiveness of reinforcement and nonreinforcement. In: Prokasy, W.E., Black, A.H. (Eds.), Classical Conditioning II: Current Research and Theory. Appleton- CenturyCrofts, New York, pp. 64-99.

Rumelhart, D.E., Hinton, G.E., McClelland, J.L., 1986. A general framework for parallel distributed processing. In: Rumelhart, D.E., McClelland, J.L. (Eds.), Parallel Distributed Processing: Explorations in the Microstructure of Cognition Vol. 1. MIT Press, Cambridge, MA, pp. 45-76.

Seidenberg, M.S., 1990. Lexical access: another theoretical soupstone? In: Balota, D.A., Flores d'Arcais, G.B., Rayner, K. (Eds.), Comprehension Processes in Reading. Routledge, New York, pp. 33-71.

Seidenberg, M.S., McClelland, J.L., 1989. A distributed developmental model of word recognition and naming. Psychol. Rev. 96 (4), 523-568.

Spitzer, M., Braun, U., Hermle, L., Maier, S., 1993. Associative semantic network dysfunction in thought-disordered schizophrenic patients: direct evidence from indirect semantic priming. Biol. Psychiatry 34, 864-877. https://doi.org/10.1016/00063223(93)90054-H.

Spitzer, R.L., Williams, J.B., Gibbon, M., First, M.B., 1992. The structured clinical Interview for DSM-III-R (SCID). I: history, rationale, and description. Arch. Gen. Psychiatry 49 (8), 624-629. https://doi.org/10.1001/archpsyc.1992. 01820080032005.

Swaab, T.Y., Boudewyn, M.A., Long, D.L., Luck, S.J., Kring, A.M., Ragland, J.D., Carter, C.S., 2013. Spared and impaired spoken discourse processing in schizophrenia: effects of local and global language context. J. Neurosci. 33 (39), 15578-15587. https://doi. org/10.1523/jneurosci.0965-13.2013.

Thompson-Schill, S.L., Bedny, M., Goldberg, R.F., 2005. The frontal lobes and the regulation of mental activity. Curr. Opin. Neurobiol. 15 (2), 219-224. https://doi.org/ 10.1016/j.conb.2005.03.006.

Tzourio-Mazoyer, N., Landeau, B., Papathanassiou, D., Crivello, F., Etard, O., Delcroix, N., Joliot, M., 2002. Automated anatomical labeling of activations in SPM using a macroscopic anatomical parcellation of the MNI MRI single-subject brain. NeuroImage 15 (1), 273-289. https://doi.org/10.1006/nimg.2001.0978.

Uusitalo, M.A., Ilmoniemi, R.J., 1997. Signal-space projection method for separating MEG or EEG into components. Med. Biol. Eng. Comput. 35 (2), 135-140. https://doi.org/ 10.1007/Bf02534144.

Vinogradov, S., Ober, B.A., Shenaut, G.K., 1992. Semantic priming of word pronunciation and lexical decision in schizophrenia. Schizophr. Res. 8, 171-181. https://doi.org/ 10.1016/0920-9964(92)90033-2.

Weber, K., Lau, E.F., Stillerman, B., Kuperberg, G.R., 2016. The Yin and the Yang of prediction: an fMRI study of semantic predictive processing. PLoS One 11 (3). 〈http://dx.doi.org/ARTNe014863710.1371/journal.pone.0148637〉.

Wheatley, T., Weisberg, J., Beauchamp, M.S., Martin, A., 2005. Automatic priming of semantically related words reduces activity in the fusiform gyrus. J. Cogn. Neurosci. 17 (12), 1871-1885.

Wilson, L.B., Rojas, D.C., Shatti, S., Tregellas, J.R., 2013. Greater neuronal responses during automatic semantic processing in schizophrenia. NeuroReport 24 (5), 212. https://doi.org/10.1097/WNR.0b013e32835eb688.

Worsley, K.J., Marrett, S., Neelin, P., Vandal, A.C., Friston, K.J., Evans, A.C., 1996. A unified statistical approach for determining significant signals in images of cerebral activation. Hum. Brain Mapp. 4 (1), 58-73. https://doi.org/10.1002/(sici)1097. 0193(1996)4:1 < 58::aid-hbm4 > 3.0.co;2-o. 